\title{
ORBIT COHERENCE IN PERMUTATION GROUPS
}

\author{
JOHN R. BRITNELL AND MARK WILDON
}

\begin{abstract}
This paper introduces the notion of orbit coherence in a permutation group. Let $G$ be a group of permutations of a set $\Omega$. Let $\pi(G)$ be the set of partitions of $\Omega$ which arise as the orbit partition of an element of $G$. The set of partitions of $\Omega$ is naturally ordered by refinement, and admits join and meet operations. We say that $G$ is join-coherent if $\pi(G)$ is join-closed, and meet-coherent if $\pi(G)$ is meet-closed.

Our central theorem states that the centralizer in $\operatorname{Sym}(\Omega)$ of any permutation $g$ is meet-coherent, and subject to a certain finiteness condition on the orbits of $g$, also join-coherent. In particular, if $\Omega$ is a finite set then the orbit partitions of elements of the centralizer in $\operatorname{Sym}(\Omega)$ of $g$ form a lattice.

A related result states that the intransitive direct product and the imprimitive wreath product of two finite permutation groups are joincoherent if and only if each of the groups is join-coherent. We also classify the groups $G$ such that $\pi(G)$ is a chain and prove two further theorems classifying the primitive join-coherent groups of finite degree and the join-coherent groups of degree $n$ normalizing a subgroup generated by an $n$-cycle.
\end{abstract}

\section{INTRODUCTION}

Let $G$ be a group acting on a set $\Omega$. Each element $g$ of $G$ has associated with it a partition $\pi(g)$ of $\Omega$, whose parts are the orbits of $g$. We define $\pi(G)$ to be the set $\{\pi(g) \mid g \in G\}$.

If $\mathcal{P}, \mathcal{Q}$ are two partitions of $\Omega$ then we say that $\mathcal{P}$ is a refinement of $\mathcal{Q}$, and write $\mathcal{P} \preccurlyeq \mathcal{Q}$, if every part of $\mathcal{P}$ is contained in a part of $\mathcal{Q}$. The set of all partitions of $\Omega$ forms a lattice under $\preccurlyeq$. That is to say, any two partitions $\mathcal{P}$ and $\mathcal{Q}$ have a supremum with respect to refinement, denoted $\mathcal{P} \vee \mathcal{Q}$, and an infimum with respect to refinement, denoted $\mathcal{P} \wedge \mathcal{Q}$. The parts of $\mathcal{P} \wedge \mathcal{Q}$ are precisely the non-empty intersections of the parts of $\mathcal{P}$ and $\mathcal{Q}$; for a description of $\mathcal{P} \vee \mathcal{Q}$ see Section 2 below. The lattice of all partitions of $\Omega$ is called the congruence lattice on $\Omega$, and is denoted $\operatorname{Con}(\Omega)$.

The object of this paper is to prove a number of interesting structural and classification results on the permutation groups possessing one or both of the following properties.

2010 Mathematics Subject Classification. 20B10; (secondary) 20E22, 06A12. 
Definition. Let $G$ be a group acting on a set.

(1) We say that $G$ is join-coherent if $\pi(G)$ is closed under $\vee$.

(2) We say that $G$ is meet-coherent if $\pi(G)$ is closed under $\wedge$.

We refer to these properties collectively as orbit coherence properties. Our first main theorem describes the groups $G$ such that $\pi(G)$ is a chain. It is clear that any such group is both join- and meet-coherent.

Theorem 1. Let $\Omega$ be a set, and let $G \leq \operatorname{Sym}(\Omega)$ be such that $\pi(G)$ is a chain. There is a prime $p$ such that the length of any cycle of any element of $G$ is a power of $p$. Furthermore, $G$ is abelian, and either periodic or torsion-free.

(a) If $G$ is periodic then $G$ is either a finite cyclic group of p-power order, or else isomorphic to the Prüfer p-group.

(b) If $G$ is torsion-free then $G$ is isomorphic to a subgroup of the p-adic rational numbers $\mathbf{Q}_{p}$. In this case $G$ has infinitely many orbits on $\Omega$, and the permutation group induced by its action on any single orbit is periodic.

Our second theorem determines when a direct product or wreath product of permutation groups inherits join coherence from its factors. The actions of these groups referred to in this theorem are defined in Sections 4 and 5 below.

Theorem 2. Let $X$ and $Y$ be sets and let $G \leq \operatorname{Sym}(X)$ and $H \leq \operatorname{Sym}(Y)$ be permutation groups.

(a) If $G$ and $H$ are finite then $G \times H$ is join-coherent in its product action on $X \times Y$ if and only if $G$ and $H$ are join-coherent and have coprime orders.

(b) If $Y$ is finite then $G \imath H$ is join-coherent in its imprimitive action on $X \times Y$ if and only if $G$ and $H$ are join-coherent.

Our third main theorem, on centralizers in a symmetric group, is the central result of this paper.

Theorem 3. Let $\Omega$ be a set, let $G=\operatorname{Sym}(\Omega)$, and let $g \in G$. For $k \in$ $\mathbf{N} \cup\{\infty\}$ let $n_{k}$ be the number of orbits of $g$ of size $k$.

(a) $\operatorname{Cent}_{G}(g)$ is meet-coherent.

(b) If $n_{k}$ is finite for all $k \neq 1$, including $k=\infty$, then $\operatorname{Cent}_{G}(g)$ is joincoherent.

We also show that if the condition on the values $n_{k}$ in the second part of the theorem fails for a permutation $g \in \operatorname{Sym}(\Omega)$, then the centralizer in $\operatorname{Sym}(\Omega)$ of $g$ is not join-coherent. Therefore this condition is necessary. 
Theorem 3 implies, in particular, that any centralizer in a finite symmetric group is both join- and meet-coherent. This is a remarkable fact, and the starting point of our investigation, at least chronologically. The observation that this important class of groups exhibits orbit coherence justifies our study of these properties, and motivates the search for further examples.

The second part of the paper contains a partial classification of finite transitive join-coherent permutation groups. Our analysis depends on the fact that such a group necessarily contains a full cycle, since the join of all the orbit partitions of elements of a transitive permutation group is the trivial one-part partition. The primitive permutation groups containing full cycles are known; we use this classification to prove the following theorem.

Theorem 4. A primitive permutation group of finite degree is join-coherent if and only if it is a symmetric group or a subgroup of $\mathrm{AGL}_{1}\left(\mathbf{F}_{p}\right)$ in its action on $p$ points, where $p$ is prime.

We also give a complete classification of the finite transitive join-coherent groups in which the subgroup generated by a full cycle is normal.

Theorem 5. Let $G$ be a permutation group on $n$ points, containing a normal cyclic subgroup of order $n$ acting regularly. Let $n$ have prime factorization $\prod_{i} p_{i}^{a_{i}}$. Then $G$ is join-coherent if and only if there exists for each $i$ a transitive permutation group $G_{i}$ on $p_{i}^{a_{i}}$ points, such that:

- if $a_{i}>1$ then $G_{i}$ is either cyclic or the extension of a cyclic group of order $p_{i}^{a_{i}}$ by the automorphism $x \mapsto x^{r}$ where $r=p_{i}^{a_{i}-1}+1$,

- if $a_{i}=1$ then $G_{i}$ is a subgroup of the Frobenius group of order $p(p-1)$,

- the orders of the groups $G_{i}$ are mutually coprime,

- $G$ is permutation isomorphic to the direct product of the groups $G_{i}$ in its product action.

Note that the permutation groups classified by Theorem 5 are always imprimitive, unless $n$ is prime. It would be interesting, but we believe difficult, to extend our results to a complete classification of all finite transitive joincoherent permutation groups. The principal obstruction to such a result is the apparently hard problem of classifying those transitive join-coherent imprimitive permutation groups that do not admit a non-trivial factorization as a direct product or a wreath product, in the manner described in Theorem 2, and which do not normalize a full cycle. One example of such a group is the permutation group of degree 12 generated by

$$
\text { (17)(4 10), (1234567891011 12). }
$$


It is not hard to check this group is an imprimitive join-coherent subgroup of index 4 in $C_{4} \succ C_{3}$ and that it does not factorize as a direct product or a wreath product.

In smaller degrees our results do yield a complete classification: every join-coherent permutation group of degree at most 11 is either a cyclic group acting regularly, a symmetric group, one of the groups described in Theorem [5, or an imprimitive wreath product of join-coherent groups of smaller degree, as in Theorem 2(b).

The fact that there are no further join-coherent groups of degree at most 11, and also the join-coherence of the group of degree 12 presented above, have been verified computationally. In Section 8, and again in Section 9. we require computer calculations to verify that particular groups are not join-coherent. All of our computations have been performed using Magma [8]. The code for these computations is available from the second author's website 1

Further remarks and background. It will be clear from the statement of our main results that the majority of them concern join-coherence rather than meet-coherence. In part this is because a finitely generated transitive join-coherent permutation group must contain a full cycle, and the restriction on the structure of the group that this imposes is very useful. However an alternative characterization of join-coherence suggests that it is a particularly natural property to study: a permutation group $G$ is join-coherent if and only if for every finitely-generated subgroup $H$ of $G$, there exists an element $h \in G$ whose orbits are the same as the orbits of $H$. There is no similar characterization of meet-coherence in terms of subgroups.

There are groups which exhibit any combination of the properties of joinand meet-coherence. Any symmetric group is both join- and meet-coherent, but any non-cyclic alternating group is neither. The group $C_{2} \times C_{2}$ acting regularly on itself is meet- but not join-coherent. Examples of groups that are join- but not meet-coherent are less easy to find, but one can check that the non-cyclic group of order 21, in its action as a Frobenius group on 7 points, is such an example (see Section 7 for our general results on Frobenius groups).

In the context of lattices, the operations $\vee$ and $\wedge$ are dual to one another. This duality is not inherited to any great extent by the notions of join- and meet-coherence of permutation groups. An asymmetry can be observed even in the congruence lattice of all partitions of a set: compare for example the two parts of Lemma 2.2 below. For this reason, while there are some parts

\footnotetext{
${ }^{1}$ See www.ma.rhul.ac.uk/ uvah099
} 
of the paper, for example Section 4, where join- and meet-coherence admit a common treatment, it is usually necessary to treat each property separately.

Literature on the orbit partitions of permutations is surprisingly sparse. As we hope that this paper shows, there are interesting general properties that remain to be discovered, and we believe that further study is warranted. One earlier investigation which perhaps has something of the same flavour is that of Cameron [4] into cycle-closed permutation groups. If $G$ is a permutation group on a finite set then the cycle-closure $C(G)$ is the group generated by all of the cycles of elements of $G$. Cameron proves that any group which is equal to its cycle-closure is isomorphic, as a permutation group, to a direct product of symmetric groups in their natural action and cyclic groups acting regularly, with the factors acting on disjoint sets. He also shows that if $G=G_{0}$, and $G_{i+1}=C\left(G_{i}\right)$, then $G_{4}=G_{3}$ and that there exist groups for which $G_{2} \neq G_{3}$.

There is an extensive literature on the lattice of subspaces of a finitedimensional vector space invariant under a group of linear transformations. In this context both the lattice elements and the lattice operations differ from ours, and so there is no immediate connection to our situation. Indeed, we show in Proposition 8.7 below that the general linear group $\mathrm{GL}(V)$ is never join-coherent when $\operatorname{dim} V>1$, except in the case when $V=\mathbf{F}_{2}^{2}$, in which case it acts on $V \backslash\{0\}$ as the full symmetric group. Nonetheless, there are certain parallels that it is interesting to observe. For instance, if $T$ is an invertible linear map on a $K$-vector space $V$, then by [2, Theorem 2], the lattice of invariant subspaces of $V$ is a chain if and only if $T$ is cyclic of primary type. Analogously, it follows from Theorem 1 that if $G$ is a finite permutation group then $\pi(G)$ is a chain if and only if $G$ is cyclic of prime-power order. Thus if we regard $T$ as a permutation of $V$, then $\pi(\langle T\rangle)$ is a chain if and only if $T$ has prime-power order. When $K$ has prime characteristic, this situation arises either when $T-I$ is nilpotent, or when there is a prime $p$ such that $V$ is a direct sum of subspaces on which $T$ acts as a Singer element of $p$-power order. Such elements exist, for example, in $\mathrm{GL}_{d}\left(\mathbf{F}_{2^{a}}\right)$ whenever $2^{a d}-1$ is a prime power. For an introduction to the theory of invariant subspaces we refer the reader to [2].

There are various areas of group theory in which lattices have previously arisen which are not directly related to orbit partitions. Subgroup lattices, for example, have been well studied. A well-known theorem of Ore [10] states that the subgroup lattice of a group $G$ is distributive if and only if $G$ is locally cyclic. Locally cyclic groups are also important in this paper: in 
Proposition 3.3 we show that they are precisely the groups that are joincoherent in their regular action. A $p$-group is locally cyclic if and only if it is a subgroup of the Prüfer $p$-group; these groups appear in Theorem 1, as the class of transitive permutation groups $G$ such that $\pi(G)$ is a chain.

Outline. The outline of this paper is as follows. In Section 2 we prove some general results with a lattice-theoretic flavour that will be used throughout the paper. We begin our structural results in Section 3 where we determine when a group acting regularly on itself is join- or meet-coherent. This section also contains a proof of Theorem 1 on the permutation groups $G$ for which $\pi(G)$ is a chain.

Theorem 2 is proved for direct products in Proposition 4.3, and for wreath products in Proposition 2.4 and in Section 5. Theorem 3 on centralizers is proved in Section 6. We have chosen to offer logically independent arguments in Section 5 and Section 6, even though the results in these sections are quite closely connected. This is partly so that they may be read independently, and partly because the two lines of approach appear to offer different insights.

The second part of the paper, which focusses on classification results, begins in Section 7 where we classify join-closed Frobenius groups of prime degree. In Section 8 we determine when a linear group has a join-coherent action on points or lines; these results are used in the proof of Theorem 4 in Section 9. Finally, Theorem 5 is proved in Section 10 .

To avoid having to specify common group actions every time they occur, we shall adopt the following conventions. Any group mentioned as acting on a set at its first appearance will be assumed always to act on that set, unless another action is explicitly given. In particular, $\operatorname{Sym}(\Omega)$ always acts naturally on the set $\Omega$, and the finite symmetric group $S_{n}$ always acts on $n$ points. The cyclic group $C_{k}$, for $k \in \mathbf{N}$, acts on itself by translation. All maps are written on the right.

\section{Partitions And ImPRimitive ACtions}

In this section we collect some facts about lattices of partitions that will be useful in later parts of the paper. For an introduction to the general theory, see for instance [6].

Given a set partition $\mathcal{P}$ of a set $\Omega$ we define a corresponding relation $\equiv_{\mathcal{P}}$ on $\Omega$ in which $x \equiv_{\mathcal{P}} y$ if and only if $x$ and $y$ lie in the same part of $\mathcal{P}$. If $\mathcal{P}$ and $\mathcal{Q}$ are set partitions of $\Omega$ then it is not hard to see that $\mathcal{P} \vee \mathcal{Q}$ is the set partition $\mathcal{R}$ such that $\equiv_{\mathcal{R}}$ is the transitive closure of the relation $\equiv$ defined 
on $\Omega$ by

$$
x \equiv y \Longleftrightarrow x \equiv_{\mathcal{P}} y \text { or } x \equiv_{\mathcal{Q}} y .
$$

Similarly $\mathcal{P} \wedge \mathcal{Q}$ is the set partition $\mathcal{R}$ such that

$$
x \equiv_{\mathcal{R}} y \Longleftrightarrow x \equiv_{\mathcal{P}} y \text { and } x \equiv_{\mathcal{Q}} y .
$$

Equivalently, as we have already remarked,

$$
\mathcal{P} \wedge \mathcal{Q}=\{P \cap Q \mid P \in \mathcal{P}, Q \in \mathcal{Q}, P \cap Q \neq \varnothing\} .
$$

The congruence lattice $\operatorname{Con}(\Omega)$ of all partitions of $\Omega$ is distributive, i.e. the lattice operations $\vee$ and $\wedge$ distribute over one another. In the language of lattice theory, a permutation group $G \leq \operatorname{Sym}(\Omega)$ is join-coherent if and only if $\pi(G)$ is an upper subsemilattice of $\operatorname{Con}(\Omega)$, meet-coherent if and only if $\pi(G)$ is a lower subsemilattice of $\operatorname{Con}(\Omega)$, and both join- and meet-coherent if and only if $\pi(G)$ is a sublattice of $\operatorname{Con}(\Omega)$.

Lemma 2.1. Let $L$ be a distributive lattice with respect to $\preccurlyeq$, and let $x \in L$.

(1) Define

$$
\begin{aligned}
& \mathrm{Up}(x)=\{y \in L \mid x \preccurlyeq y\}, \\
& \operatorname{Dn}(x)=\{y \in L \mid y \preccurlyeq x\} .
\end{aligned}
$$

Then $\operatorname{Up}(x)$ and $\operatorname{Dn}(x)$ are sublattices of $L$.

(2) The maps $\varphi^{x}: L \longrightarrow \mathrm{Up}(x)$ and $\varphi_{x}: L \longrightarrow \mathrm{Dn}(x)$ defined by

$$
y \varphi^{x}=y \vee x, \quad y \varphi_{x}=y \wedge x,
$$

are lattice homomorphisms.

Proof. The first part follows directly from the defining property of $\vee$ and $\wedge$, and the second part from the definition of distributivity.

Note that in part (1) of the following lemma, $\operatorname{Con}(\mathcal{B})$ is the congruence lattice on the set $\{B \mid B \in \mathcal{B}\}$ of parts of a partition $\mathcal{B}$.

Lemma 2.2. Let $\Omega$ be a set and let $\mathcal{B} \in \operatorname{Con}(\Omega)$.

(1) $\operatorname{Up}(\mathcal{B}) \cong \operatorname{Con}(\mathcal{B})$.

(2) $\operatorname{Dn}(\mathcal{B}) \cong \prod_{B \in \mathcal{B}} \operatorname{Con}(B)$.

Proof. If $\mathcal{B} \preccurlyeq \mathcal{A}$ then each part of $\mathcal{A}$ is a union of parts of $\mathcal{B}$. Hence $\mathcal{A}$ determines and is determined by a partition of $\mathcal{B}$; this gives a bijection between $\operatorname{Up}(\mathcal{B})$ and $\operatorname{Con}(\mathcal{B})$ that is a lattice isomorphism. For the second part we note that whenever $\mathcal{A} \preccurlyeq \mathcal{B}$, each part $B$ of $\mathcal{B}$ is a union of parts of $\mathcal{A}$, and so a subset of the parts of $\mathcal{A}$ form a partition of $B$. Clearly $\mathcal{A}$ itself is determined by these partitions of the parts of $\mathcal{B}$, and thus $\mathcal{A}$ determines 
and is determined by an element of $\prod_{B \in \mathcal{B}} \operatorname{Con}(B)$. It is easy to see that this bijection is a lattice isomorphism.

The next proposition is a straightforward consequence of Lemma 2.2, As a standing convention, we avoid the use of the word 'respectively' when the same short statement or proof works for either a join- or a meet-coherent group.

Proposition 2.3. Let $G$ be a join- or meet-coherent permutation group on $\Omega$.

(1) Let $\mathcal{B}$ be a partition of $\Omega$, and let $H$ be the group of permutations which fix every part of $\mathcal{B}$ set-wise. Then $G \cap H$ is join- or meet-coherent.

(2) Let $X \subseteq \Omega$, and let $H$ be the set-stabilizer of $X$ in $G$. Then $H$ is joinor meet-coherent.

(3) Any point-stabilizer in $G$ is join- or meet-coherent.

Proof. If $h \in \operatorname{Sym}(\Omega)$ then $\pi(h) \preccurlyeq \mathcal{B}$ if and only if $B h=B$ for all $B \in \mathcal{B}$. Thus $\pi(H)=\operatorname{Dn}(\mathcal{B})$, which is closed under $\vee$ and $\wedge$. The first part of proposition follows, since the intersection of two join- or meet-closed sets is join- or meet-closed. The second part follows from the first by taking $\mathcal{B}=\{X, \Omega \backslash X\}$, and the third part follows from the second by taking $X$ to be a singleton set.

If $G$ is a permutation group acting on a set $\Omega$, then we may consider the natural action of $G$ on $\operatorname{Con}(\Omega)$, defined for $g \in G$ and $\mathcal{P} \in \operatorname{Con}(\Omega)$ by $\mathcal{P}^{g}=\left\{P^{g} \mid P \in \mathcal{P}\right\}$. This action will be used in Lemma 6.2 below, which is the critical step in the proof of Theorem 3 ,

Recall that a transitive permutation group $G$ on $\Omega$ is said to be $\mathrm{im}$ primitive if it stabilizes a non-trivial partition $\mathcal{B}$ of $\Omega$, in the sense that $x g \equiv_{\mathcal{B}} y g \Longleftrightarrow x \equiv_{\mathcal{B}} y$ for all $x, y \in \Omega$ and $g \in G$. An equivalent restatement, using the action just defined, is that $\mathcal{B}^{g}=\mathcal{B}$ for each $g \in G$. In this case, one says that $\mathcal{B}$ is a system of imprimitivity for the action of $G$ on $\Omega$. Otherwise $G$ is primitive. If $\mathcal{B}$ is a $G$-invariant partition of $\Omega$ then $\mathcal{B}$ inherits an action of $G$, since for $B \in \mathcal{B}$ and $g \in G$ we have $B^{g} \in \mathcal{B}$.

The next proposition gives the easier direction in Theorem 2 (b). The proof of this theorem is completed in Proposition 5.2 .

Proposition 2.4. Let $G$ be a join- or meet-coherent permutation group on $\Omega$, and let $\mathcal{B}$ be a system of imprimitivity for the action.

(1) The action of $G$ on $\mathcal{B}$ is join- or meet-coherent.

(2) The set-stabilizer in $G$ of a part $B$ of $\mathcal{B}$ acts join-or meet-coherently on $B$. 
Proof. The second part is immediate from Proposition 2.3(2). For the first part, let $\vartheta: \operatorname{Up}(\mathcal{B}) \longrightarrow \operatorname{Con}(\mathcal{B})$ be the isomorphism in Lemma 2.2(1). By Lemma $2.1(2)$, the composite map $\varphi^{\mathcal{B}} \vartheta: \operatorname{Con}(\Omega) \longrightarrow \operatorname{Con}(\mathcal{B})$ is a homomorphism. It is easy to check that if $g \in G$ has orbit partition $\mathcal{P}$ on $\Omega$ then $g$ has orbit partition $\mathcal{P} \varphi^{\mathcal{B}} \vartheta=(\mathcal{P} \vee \mathcal{B}) \vartheta$ on $\mathcal{B}$. The result now follows from the fact that $\operatorname{Con}(\mathcal{B})$ is distributive.

\section{REgular REPRESENTATIONS AND CHAins}

Let $G$ be a group of permutations of a set $\Omega$. We say that $G$ acts semiregularly if every element of $\Omega$ has a trivial point stabilizer in $G$. This is equivalent to the condition that for every element $g \in G$, all of the parts of the orbit partition $\pi(g)$ of $\Omega$ are of the same size. We say that the action of $G$ is regular if it is semiregular and transitive.

Proposition 3.1. A group $G$ acting semiregularly is meet-coherent.

Proof. Suppose that $G$ acts semiregularly on $\Omega$. Let $x, y \in G$, and let $z$ be a generator for the cyclic group $\langle x\rangle \cap\langle y\rangle$. We shall show that $\pi(z)=$ $\pi(x) \wedge \pi(y)$.

Let $u \in \Omega$, and let $P$ and $Q$ be the parts of $\pi(x)$ and $\pi(y)$ respectively which contain $u$. Then

$$
P=\left\{u x^{i} \mid i \in \mathbf{Z}\right\} \quad \text { and } \quad Q=\left\{u y^{j} \mid j \in \mathbf{Z}\right\} .
$$

Let $v \in P \cap Q$, and let $i, j \in \mathbf{Z}$ be such that $v=u x^{i}=u y^{j}$. By the semiregularity of $G$, we have $x^{i}=y^{j}$, and so $x^{i}, y^{j} \in\langle z\rangle$. It follows that $P \cap Q=\left\{u z^{k} \mid k \in \mathbf{Z}\right\}$, and hence that $P \cap Q$ is a part of $\pi(z)$.

We recall that a group $G$ is said to be locally cyclic if any pair of elements of $G$ generate a cyclic subgroup. The following lemma states a well-known fact.

Lemma 3.2. A group is locally cyclic if and only if it is isomorphic to a section of $\mathbf{Q}$.

Proof. It is clear that a locally cyclic group is either periodic or torsionfree. If it is periodic then it has at most one subgroup of order $n$ for each $n \in \mathbf{N}$, and it is easy to see that it embeds into the quotient group $\mathbf{Q} / \mathbf{Z}$; for torsion-free groups the result was first proved in [1] 2

Proposition 3.3. A group $G$ acting regularly is join-coherent if and only if it is locally cyclic.

\footnotetext{
${ }^{2}$ We thank Mark Sapir for this reference.
} 
Proof. We may assume without loss of generality that $G$ is transitive, and so we may suppose that it acts on itself by translation. Let $x, y \in G$, and let $H=\langle x, y\rangle$. The partition $\pi(x) \vee \pi(y)$ is precisely the partition of $G$ into cosets of $H$. If $H=\langle z\rangle$ then we have $\pi(x) \vee \pi(y)=\pi(z) \in \pi(G)$. On the other hand, if $H$ is not cyclic then it cannot be a part of $\pi(z)$ for any $z \in G$, and so $\pi(x) \vee \pi(y) \notin \pi(G)$.

We define a locally cyclic group of particular importance to us.

Definition. The Prüfer $p$-group, $\mathbf{P}$, is the subgroup of $\mathbf{Q} / \mathbf{Z}$ generated by the cosets containing $1 / p^{i}$ for $i \in \mathbf{N}$.

The group $\mathbf{P}$ appears in the statement of Theorem 1, whose proof occupies the remainder of this section. We begin with the following proposition.

Proposition 3.4. Let $\Omega$ be a set, and let $G \leq \operatorname{Sym}(\Omega)$ be such that $\pi(G)$ is a chain.

(1) No element of $G$ has an infinite cycle.

(2) Let $\mathcal{O}$ be an orbit of $G$, and let $G_{\mathcal{O}} \leq \operatorname{Sym}(\mathcal{O})$ be the permutation group induced by the action of $G$ on $\mathcal{O}$. Then $G_{\mathcal{O}}$ acts regularly on $\mathcal{O}$.

(3) There is a prime $p$ such that every cycle of every element of $g$ has p-power length.

(4) If $G$ acts transitively, then there is a prime $p$ such that $G$ is isomorphic to a subgroup of the Prüfer p-group $\mathbf{P}$.

Proof. It is clear that if $g \in G$ has an infinite cycle then $\pi\left(g^{a}\right)$ and $\pi\left(g^{b}\right)$ are incomparable whenever $a$ and $b$ are natural numbers such that neither divides the other. Clearly this implies (1).

For (2), let $g$ be an element of $G$ which acts non-trivially on $\mathcal{O}$, and suppose that $g$ has a fixed point $x \in \mathcal{O}$. Let $z \in \mathcal{O}$ be such that $z g \neq z$, and let $h \in G$ be such that $x h=z$. Then $g^{h}$ has $z$ as a fixed point, and $x g^{h} \neq x$. Hence the partitions $\pi(g)$ and $\pi\left(g^{h}\right)$ are incomparable, a contradiction. Therefore $g$ has no fixed points on $\mathcal{O}$, and it follows that the action of $G_{\mathcal{O}}$ is regular.

For (3), suppose that there exist distinct primes $q$ and $r$ such that $\pi(g)$ has a part $Q$ of size divisible by $q$, and another part $R$ of size divisible by $r$. Let $m$ be the order of the permutation induced by $g$ on $Q \cup R$. Then $\pi\left(g^{m / r}\right)$ has singleton parts corresponding to the elements of $Q$, and $R$ is a union of parts of $\pi\left(g^{m / r}\right)$ of size at least 2. A similar remark holds for $\pi\left(g^{m / q}\right)$ with $Q$ and $R$ swapped, and so $\pi\left(g^{m / q}\right)$ and $\pi\left(g^{m / r}\right)$ are not comparable; again this is a contradiction. It follows that for each $g \in G$, there is a prime $p_{g}$ such that every cycle of $g$ has length a power of $p_{g}$. 
We now show that $p_{g}=p_{h}$ for all non-identity permutations $g, h \in G$. We may suppose without loss of generality that $\pi(h) \preccurlyeq \pi(g)$, and so each orbit of $\langle g\rangle$ is a union of orbits of $\langle h\rangle$. But by (2), $h$ acts regularly on each of its orbits. There exists a $\langle g\rangle$-orbit $\mathcal{O}$ on which $h$ acts non-trivially, and so $p_{h}$ divides $|\mathcal{O}|$. But $|\mathcal{O}|$ is a power of $p_{g}$, and so $p_{h}=p_{g}$.

For (4), we note that if $G$ is transitive, then by (2) it acts regularly. By Proposition 3.3, $G$ is locally cyclic. By Lemma 3.2 it follows that $G$ is isomorphic to a section of $\mathbf{Q}$. Now an element $g \in G$ has only finite cycles by (1), and since $G$ acts regularly, all of the cycles of $g$ have the same length. Therefore $g$ has finite order, and so $G$ is not torsion-free. Hence $G$ is isomorphic to a subgroup of $\mathbf{Q} / \mathbf{Z}$. Write $g$ as $a / b+\mathbf{Z}$, where $a$ and $b$ are coprime. Since every cycle of $g$ has $p$-power length by (3), the denominator $b$ must be a power of $p$; hence $G$ is isomorphic to a subgroup of the Prüfer p-group.

We note that any subgroup of $\mathbf{P}$ is either cyclic of $p$-power order, or equal to $\mathbf{P}$ itself. Therefore (4) implies that any finite group whose orbit partitions form a chain is cyclic of prime-power order.

There are interesting examples of groups $G$ acting intransitively on an infinite set, such that $G$ is not locally cyclic, but $\pi(G)$ is a chain. Let $\alpha$ be an irrational element of the $p$-adic integers $\mathbf{Z}_{p}$, such that $p$ does not divide $\alpha$, and define $\alpha_{i} \in\left\{0,1, \ldots, p^{i}-1\right\}$ by $\alpha_{i}=\alpha \bmod p^{i}$. For instance, we may take $p=2$ and $\alpha=1010010001000010 \ldots \in \mathbf{Z}_{2}$; the sequence $\left(\alpha_{i}\right)$ here is

$$
(1,1,5,5,5,37,37,37,37,549, \ldots)
$$

Let $\Omega$ be an infinite set and let $\left\{c_{i} \mid i \in \mathbf{N}\right\}$ be a set of mutually disjoint cycles in $\operatorname{Sym}(\Omega)$, such that $c_{i}$ has length $p^{i}$. Let $G \leq \operatorname{Sym}(\Omega)$ be generated by $g$ and $h$, where $g=\prod_{i=1}^{\infty} c_{i}$ and $h=\prod_{i=1}^{\infty} c_{i}^{\alpha_{i}}$. Then $g$ and $h$ have the same orbit partition, but it is easily seen that they are not powers of one another. Hence $G$ is not cyclic. However, the permutation group induced by $G$ on any finite set of its orbits is cyclic, since for any given $j$ there exists $\beta_{j} \in \mathbf{N}$ such that $\alpha_{j} \beta_{j} \equiv 1 \bmod p^{j}$, and so $h^{\beta_{j}}$ agrees with $g$ on the orbits of all of the cycles $c_{i}$ for $i \leq j$. It follows easily that $\pi(G)$ is a chain.

The group in this example falls under (2) in Theorem 1, to prove this theorem we shall use Proposition 3.4 and the following lemma 3

\footnotetext{
${ }^{3}$ The authors would like to thank Benjamin Klopsch and John MacQuarrie for helpful conversations on this subject.
} 
Lemma 3.5. Let $p$ be a prime and let $I$ be a totally ordered set. For each $i \in I$, let $P_{i}$ be an isomorphic copy of the Prüfer p-group $\mathbf{P}$. Let

$$
\left\{f_{j i}: P_{j} \rightarrow P_{i} \mid i, j \in I, i \leq j\right\}
$$

be a set of non-zero homomorphisms, with the property that $f_{k j} f_{j i}=f_{k i}$ whenever $i \leq j \leq k$. Let $M$ be the inverse limit $\varliminf_{\longleftarrow} P_{i}$, taken with respect to the totally ordered set $I$ and the homomorphisms $f_{j i}$. If all but finitely many of the $f_{j i}$ are isomorphisms then $M \cong \mathbf{P}$, and otherwise $M \cong \mathbf{Q}_{p}$, the additive group of p-adic rational numbers.

Proof. If $f: \mathbf{P} \rightarrow \mathbf{P}$ is an endomorphism, then for each $i \in \mathbf{N}$, there exists a unique $a_{i} \in\left\{0,1, \ldots, p^{i}-1\right\}$ such that

$$
\left(x / p^{i}+\mathbf{Z}\right) f=a_{i}\left(x / p^{i}+\mathbf{Z}\right) \quad \text { for all } x \in \mathbf{Z} .
$$

It is easily seen that if $i \leq j$ then $a_{j} \equiv a_{i} \bmod p^{i}$. Therefore if $a \in \mathbf{Z}_{p}$ is the $p$-adic integer such that $a \equiv a_{i} \bmod p^{i}$ for each $i \in \mathbf{N}$, then $f$ is the map $\mu(a): \mathbf{P} \rightarrow \mathbf{P}$ defined by $(\star)$ above. It follows that at most a countable infinity of the maps $f_{j i}$ are non-isomorphisms.

Observe that $\mu(a)$ is surjective unless $a=0$, and an isomorphism if and only if $a$ is not divisible by $p$. If all but finitely many of the $f_{j i}$ are isomorphisms, then it is clear that $M$ is isomorphic to $\mathbf{P}$. Otherwise there exists an infinite increasing sequence $\left(i_{k}\right)$ of elements of $I$, such that if we set $R_{k}=P_{i_{k}}$ and

$$
g_{k}=f_{i_{k+1} i_{k}}: R_{k+1} \rightarrow R_{k}
$$

for $k \in \mathbf{N}$, then each $g_{k}$ is a non-isomorphism, and $M \cong \lim _{\longleftarrow} R_{k}$.

For each $k \in \mathbf{N}$, let $a_{k} \in \mathbf{Z}_{p}$ be such that $g_{k}=\mu\left(a_{k}\right)$. Let $a_{k}=p^{e_{k}} b_{k}$ where $p$ does not divide $b_{k}$; by assumption $e_{k} \geq 1$ for each $k$. Let $d_{k}=$ $\prod_{i=1}^{k-1} b_{i}$ for each $k \in \mathbf{N}$. Then in the commutative diagram

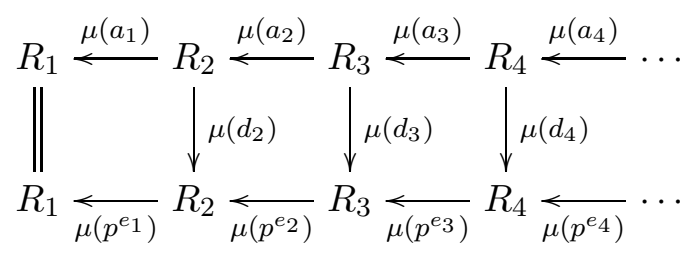

all of the vertical arrows are isomorphisms. It follows easily that the inverse limits constructed with respect to the top and bottom rows are isomorphic. Moreover, the inverse system

$$
P_{1} \stackrel{\mu(p)}{\longleftarrow} P_{2} \stackrel{\mu(p)}{\longleftarrow} P_{3} \stackrel{\mu(p)}{\longleftarrow} P_{4} \stackrel{\mu(p)}{\longleftarrow} \cdots
$$

in which all of the maps are multiplication by $p$ is a refinement of the bottom row of the diagram $(\dagger)$, and so it defines the same inverse limit. 
Finally we note that $\mathbf{P} \cong \mathbf{Q}_{p} / \mathbf{Z}_{p}$, and that after applying this isomorphism, the map $\mu(p): \mathbf{P} \rightarrow \mathbf{P}$ is induced by multiplication by $p$ in $\mathbf{Q}_{p}$. Hence $M \cong \lim \mathbf{Q}_{p} / p^{k} \mathbf{Z}_{p}$. Since $p^{k} \mathbf{Z}_{p}$ is an open subgroup of $\mathbf{Q}_{p}$, and since $\bigcap_{k} p^{k} \mathbf{Z}_{p}=\{0\}$, it follows that $M \cong \mathbf{Q}_{p}$ as required.

We are now ready to prove Theorem 1, which we restate below for convenience.

Theorem 1. Let $\Omega$ be a set, and let $G \leq \operatorname{Sym}(\Omega)$ be such that $\pi(G)$ is a chain. There is a prime $p$ such that the length of any cycle of any element of $G$ is a power of $p$. Furthermore, $G$ is abelian, and either periodic or torsion-free.

(a) If $G$ is periodic then $G$ is either a finite cyclic group of p-power order, or else isomorphic to the Prüfer p-group.

(b) If $G$ is torsion-free then $G$ is isomorphic to a subgroup of the p-adic rational numbers $\mathbf{Q}_{p}$. In this case $G$ has infinitely many orbits on $\Omega$, and the permutation group induced by its action on any single orbit is periodic.

Proof. By Proposition 3.4(4), there is a prime $p$ such that $G$ acts as a subgroup of the Prüfer $p$-group $\mathbf{P}$ on each of its orbits. It follows that $G$ is abelian. Suppose that $g \in G$ has infinite order, and $h \in G$ has finite order $p^{a}$. Then $\pi(h) \prec \pi\left(g^{i}\right)$ for any $i \in \mathbf{N}$, since $g$ has cycles of length greater than $i p^{a}$. But each cycle of $g$ is finite, and so every element of $\Omega$ is fixed by some power of $g$. Hence $h$ is the identity of $G$. This shows that $G$ is either torsion-free or periodic.

Suppose that $K_{1}$ and $K_{2}$ are the kernels of the action of $G$ on distinct orbits. If there exists $k_{1} \in K_{1} \backslash K_{2}$ and $k_{2} \in K_{2} \backslash K_{1}$ then the orbit partitions $\pi\left(k_{1}\right)$ and $\pi\left(k_{2}\right)$ are clearly incomparable. Hence the kernels of the action of $G$ on its various orbits form a chain of subgroups of $G$. Since $G$ acts faithfully on $\Omega$, the intersection of all these kernels is trivial.

Suppose that $G$ is periodic. Let $g_{1}, \ldots, g_{r} \in G$ be a finite collection of elements, and let $H=\left\langle g_{1}, \ldots, g_{r}\right\rangle$. Then $H$ is finite. Since $H$ satisfies the descending chain condition on subgroups, there is an orbit $\mathcal{O}$ of $G$ for which the kernel $K_{\mathcal{O}}$ of $G$ acting on $\mathcal{O}$ intersects trivially with $H$. Therefore $H$ is isomorphic to a subgroup of $G / K_{\mathcal{O}}$. It now follows from Proposition 3.4(4) that $H$ is isomorphic to a subgroup of $\mathbf{P}$. Hence any finitely generated subgroup of $G$ is cyclic, and so $G$ is locally cyclic. Now by Lemma 3.2 we see that $G$ itself is isomorphic to a subgroup of $\mathbf{P}$.

The remaining case is when $G$ is torsion-free. Let $\mathcal{O}_{i}$ for $i \in I$ be the set of orbits of $G$, where $I$ is a suitable indexing set, and let $K_{i}$ be the 
kernel of $G$ acting on $\mathcal{O}_{i}$. Order $I$ so that for $i, j \in I$, we have $i \leq j$ if and only if $K_{i} \geq K_{j}$. For $i \leq j$ let $f_{j i}: G / K_{j} \rightarrow G / K_{i}$ be the canonical surjection. Fix for each $i \in I$ an isomorphism $G / K_{i} \rightarrow P_{i}$ where $P_{i} \cong \mathbf{P}$. Since $\bigcap_{i \in I} K_{i}=\left\{1_{G}\right\}$ and each $G / K_{i}$ is isomorphic to a subgroup of $\mathbf{P}$, we see that $G$ is isomorphic to a subgroup of the inverse $\operatorname{limit} \lim _{P_{i}}$, taken with respect to the totally ordered set $I$ and the homomorphisms $f_{j i}$. The theorem now follows from Lemma 3.5 .

We end this section by remarking that if $G \leq \operatorname{Sym}(\Omega)$ is isomorphic to a subgroup of $\mathbf{P}$, then it has an orbit on $\Omega$ on which it acts faithfully and regularly. For if $G$ is non-trivial then the intersection of the non-trivial subgroups of $G$ has order $p$; now since $G$ acts faithfully on $\Omega$, it follows that there is an orbit $\mathcal{O}$ on which $G$ acts with trivial kernel. Since $G$ is abelian, its action on $\mathcal{O}$ is regular.

\section{Direct products}

Suppose that $G$ and $H$ are groups acting on sets $X$ and $Y$ respectively. There are two natural actions of the direct product $G \times H$, namely the intransitive action on the disjoint union $X \cup Y$ and the product action on $X \times Y$. For $(g, h) \in G \times H$ the intransitive action is defined by

$$
z(g, h)= \begin{cases}z g & \text { if } z \in X \\ z h & \text { if } z \in Y\end{cases}
$$

where $z \in X \cup Y$, and the product action is defined by $(x, y)(g, h)=(x g, y h)$ where $(x, y) \in X \times Y$. The product action is the subject of Theorem 2(a), which we prove in this section. Both of these actions also arise in later parts of the paper.

Lemma 4.1. Let $X$ and $Y$ be sets. Let $g \in \operatorname{Sym}(X)$ and $h \in \operatorname{Sym}(Y)$, and let $k=(g, h) \in \operatorname{Sym}(X) \times \operatorname{Sym}(Y)$.

(1) In its action on $X \cup Y$ we have $\pi(k)=\pi(x) \cup \pi(y)$.

(2) Suppose that $g$ and $h$ have finite coprime orders. Then in its action on $X \times Y$ we have $\pi(k)=\{P \times Q \mid P \in \pi(g), Q \in \pi(h)\}$.

Proof. Both parts are straightforward.

The intransitive action of a direct product is dealt with in the next proposition. 
Proposition 4.2. Let $G$ and $H$ be groups acting on sets $X$ and $Y$ respectively.

(1) The action of $G \times H$ on $X \cup Y$ is join-coherent if and only if the actions of $G$ and $H$ are join-coherent.

(2) The action of $G \times H$ on $X \cup Y$ is meet-coherent if and only if the actions of $G$ and $H$ are meet-coherent.

Proof. Suppose that $g_{1}, g_{2}, g_{3} \in G$ and $h_{1}, h_{2}, h_{3} \in H$. It is clear from Lemma 4.1(1) that

$$
\pi\left(g_{1}, h_{1}\right) \vee \pi\left(g_{2}, h_{2}\right)=\pi\left(g_{3}, h_{3}\right)
$$

if and only if $\pi\left(g_{1}\right) \vee \pi\left(g_{2}\right)=\pi\left(g_{3}\right)$ and $\pi\left(h_{1}\right) \vee \pi\left(h_{2}\right)=\pi\left(h_{3}\right)$, and similarly

$$
\pi\left(g_{1}, h_{1}\right) \wedge \pi\left(g_{2}, h_{2}\right)=\pi\left(g_{3}, h_{3}\right)
$$

if and only if $\pi\left(g_{1}\right) \wedge \pi\left(g_{2}\right)=\pi\left(g_{3}\right)$ and $\pi\left(h_{1}\right) \wedge \pi\left(h_{2}\right)=\pi\left(h_{3}\right)$. The result follows.

Turning to the product action, we prove Theorem 2(a). For convenience we restate this result as the following proposition.

Proposition 4.3. Let $X$ and $Y$ be sets and let $G \leq \operatorname{Sym}(X)$ and $H \leq$ $\operatorname{Sym}(Y)$ be finite permutation groups. Then $G \times H$ is join-coherent on $X \times Y$ if and only if $G$ and $H$ are both join-coherent, and have coprime orders.

Proof. It is obvious that if either $G$ or $H$ is not join-coherent, then neither is $G \times H$. So we suppose that $G$ and $H$ act join-coherently. If $|G|$ and $|H|$ are coprime then the join-coherence of $G \times H$ on $X \times Y$ follows from Lemma 4.1(2).

Suppose conversely that there is a prime $p$ which divides both $|G|$ and $|H|$. Let $g \in G$ have order $p^{a}$, and let $h \in H$ have order $p^{b}$, where these are the largest orders of $p$-elements in each group. Then it is clear that $\pi(g)$ has a part of size $p^{a}$, and $\pi(h)$ a part of size $p^{b}$. Now $\pi(g, 1) \vee \pi(1, h)$ has a part of size $p^{a+b}$, and it follows that it cannot be in $\pi(G \times H)$, since the greatest order of a $p$-element in $G \times H$ is $\max \left(p^{a}, p^{b}\right)$. Hence if $G \times H$ is join-coherent then $G$ and $H$ have coprime orders.

The following result may be viewed as a partial converse to Proposition 4.3. It is used in the proof of Theorem 5 in Section 10 below.

Proposition 4.4. Suppose that $G$ acts on finite sets $X$ and $Y$, and that these actions have kernels $K_{X}$ and $K_{Y}$ respectively, where $K_{X} \cap K_{Y}=1$. If $G$ is join-coherent on $X \times Y$ then

(1) $K_{Y}$ is join-coherent on $X$ and $K_{X}$ is join-coherent on $Y$; 
(2) $\left|K_{X}\right|$ and $\left|K_{Y}\right|$ are coprime;

(3) $G=K_{Y} K_{X} \cong K_{Y} \times K_{X}$.

Proof. Consider the partition $\mathcal{B}$ of $X \times Y$ into parts $X \times\{y\}$ for $y \in Y$. Clearly $K_{Y}$ is the largest subgroup of $G$ which stabilizes the parts of $\mathcal{B}$, and its action on each part is that of $K_{Y}$ on $X$. Since $K_{X} \cap K_{Y}=1$, this action is faithful. Hence, by Proposition 2.3(1), the action of $K_{Y}$ on $X$ is join-coherent, and (1) follows.

Let $p$ be a prime, and let $p^{a}$ and $p^{b}$ be the largest powers of $p$ dividing $\left|G / K_{X}\right|$ and $\left|G / K_{Y}\right|$ respectively. If $P$ is a Sylow $p$-subgroup of $G$, then $P$ contains elements $g_{X}$ and $g_{Y}$ such that $g_{X} K_{X}$ has order $p^{a}$ in $G / K_{X}$ and $g_{Y} K_{Y}$ has order $p^{b}$ in $G / K_{Y}$. It follows that $g_{X}$ has an orbit $\mathcal{O}_{X}$ on $X$ of size $p^{a}$, and that $g_{Y}$ has an orbit $\mathcal{O}_{Y}$ on $Y$ of size $p^{b}$. Now $\left\langle g_{X}, g_{Y}\right\rangle$ is a subgroup of $P$, and so its orbits on $X \times Y$ have $p$-power order. One of these orbits contains $\mathcal{O}_{X} \times \mathcal{O}_{Y}$, and so has order $p^{c}$ for some $c \geq a+b$.

Since $G$ is join-coherent on $X \times Y$, it must contain an element $g$ whose orbits are those of $\left\langle g_{X}, g_{Y}\right\rangle$. It is clear that $g$ must be a $p$-element of order at least $p^{a+b}$. Since $G / K_{X}$ and $G / K_{Y}$ have $p$-exponents $p^{a}$ and $p^{b}$ respectively, it follows that $g^{a} \in K_{X}, g^{b} \in K_{Y}$ and so

$$
g^{\max (a, b)} \in K_{X} \cap K_{Y}=1 .
$$

Hence one of $a$ or $b$ is 0 and $G / K_{X}$ and $G / K_{Y}$ have coprime orders.

Suppose that $p^{r}$ divides $|G|$. Since at least one of $G / K_{X}$ or $G / K_{Y}$ has order coprime with $p$, we see that $p^{r}$ must divide one of $\left|K_{X}\right|$ or $\left|K_{Y}\right|$, and it follows that $|G|$ divides $\left|K_{X}\right| \cdot\left|K_{Y}\right|$. But since $K_{X} \cap K_{Y}=1$ we have $\left|K_{Y} K_{X}\right|=\left|K_{Y}\right| \cdot\left|K_{X}\right|$, and hence $G=K_{Y} K_{X}$; since $K_{X}$ and $K_{Y}$ are both normal, this implies that $G \cong K_{Y} \times K_{X}$, as stated in (3). Now (2) follows from the final sentence of the previous paragraph.

Necessary and sufficient criteria for the meet-coherence of $G \times H$ in its product action are harder to obtain. Since we shall not need any such results in later parts of the paper, we merely offer the following partial result.

Proposition 4.5. Let $X$ and $Y$ be sets and let $G \leq \operatorname{Sym}(X)$ and $H \leq$ $\operatorname{Sym}(Y)$ be meet-coherent permutation groups. If $G$ and $H$ are finite of coprime order, then $G \times H$ is meet-coherent on $X \times Y$.

Proof. This follows easily from Lemma 4.1(2).

There are examples of groups $G \leq \operatorname{Sym}(X)$ and $H \leq \operatorname{Sym}(Y)$, not of coprime order, such that $G \times H$ is meet-coherent on $X \times Y$. For instance 
if both $G$ and $H$ act semiregularly, then so does $G \times H$, and so by Proposition 3.1 we see that $G \times H$ is meet-coherent regardless of the orders of $G$ and $H$.

\section{WREATH PRODUCTS}

Given sets $S$ and $T$, we write $S^{T}$ for the set of maps from $T$ to $S$. As usual, we shall write all maps on the right. If $S$ is a group, then $S^{T}$ inherits a group structure as the direct product of $|T|$ copies of $S$; here $|T|$ may be infinite.

Throughout this section we let $G$ and $H$ be groups acting on sets $X$ and $Y$ respectively. The unrestricted wreath product $G ? H$ is defined to be the semidirect product $G^{Y} \rtimes H$, where the action of $H$ on $G^{Y}$ is given by $f h=h^{-1} \circ f$ for $f \in G^{Y}$ and $h \in H$.

There are two natural actions of $G \imath H$. In the first $G \imath H$ acts on $X^{Y}$ (see [5. Section 4.3]); in this action the wreath product does not generally inherit join- or meet-coherence from $G$ and $H$ : for instance $C_{2} \succ C_{3}$ is neither joinnor meet-coherent in this action. For this reason we shall not discuss it any further here.

The second action of the wreath product is the imprimitive action on $X \times Y$. If $f: Y \rightarrow G$ and $h \in H$ then this action is given by

$$
(x, y) f h=(x(y f), y h) \quad \text { for } x \in X, y \in Y \text {. }
$$

For $y \in Y$, let $B_{y}=X \times\{y\}$. Then $\left\{B_{y} \mid y \in Y\right\}$ is a system of imprimitivity for this action, in the sense defined after Proposition 2.3. In general $G$ ? $H$ does not inherit meet-coherence from $G$ and $H$ in the imprimitive action; for instance $S_{3} C_{2}$ is not meet-coherent. Join-coherence, however, is inherited in the case that $Y$ is finite, and the proof of this fact is the object of this section.

Definition. Let $\mathcal{P}$ be a partition of $X \times Y$.

(1) We write $\tilde{\mathcal{P}}$ for the partition of $Y$ defined by

$$
y_{1} \equiv_{\tilde{\mathcal{P}}} y_{2} \Longleftrightarrow\left(x_{1}, y_{1}\right) \equiv_{\mathcal{P}}\left(x_{2}, y_{2}\right) \text { for some } x_{1}, x_{2} \in X .
$$

(2) For $y \in Y$ we write $\mathcal{P}_{[y]}$ for the partition of $X$ defined by

$$
x_{1} \equiv_{\mathcal{P}_{[y]}} x_{2} \Longleftrightarrow\left(x_{1}, y\right) \equiv_{\mathcal{P}}\left(x_{2}, y\right) .
$$

The following lemma provides a characterization of the orbit partitions of the elements of a wreath product in its imprimitive action. For the application to Theorem 2(b) we only need the case where $Y$ is finite, which permits some simplifications to the statement and proof. 
Lemma 5.1. Let $\mathcal{P}$ be a partition of $X \times Y$. Then $\mathcal{P}$ is the orbit partition of an element of $G$ ? $H$ if and only if the following conditions hold.

(1) There exists an element $h \in H$ with orbit partition $\tilde{\mathcal{P}}$ on $Y$.

(2) For every $y \in Y$, there exists an element $g \in G$ with orbit partition $\mathcal{P}_{[y]}$ on $X$.

(3) If $y$ lies in an infinite part of $\tilde{\mathcal{P}}$ then $\mathcal{P}_{[y]}$ is the partition of $X$ into singleton sets.

(4) Whenever elements $y, z \in Y$ lie in the same part of $\tilde{\mathcal{P}}$, there exists $c \in G$ such that

$$
(x, y) \equiv_{\mathcal{P}}(x c, z) \text { for all } x \in X .
$$

Proof. Let $f \in G^{Y}$, let $h \in H$, and let $k=f h \in G \curlywedge H$. It is clear that if $\mathcal{P}$ is the orbit partition of $k$ on $X \times Y$, then $\tilde{\mathcal{P}}$ is the orbit partition of $h$ on $Y$, and so (1) is necessary. Let $t$ be a positive integer. A simple calculation shows that $k^{t}=f_{t} h^{t}$ where $f_{t} \in G^{Y}$ is defined by

$$
f_{t}: y \mapsto(y f)((y h) f) \cdots\left(\left(y h^{t-1}\right) f\right) .
$$

Hence

$$
(x, y) k^{t}=\left(x\left(y f_{t}\right), y h^{t}\right)
$$

for all $x \in X$, and it easily follows that (4) is necessary.

Suppose that $y \in Y$ lies in a finite $h$-orbit of size $m$. In this case it is not hard to see that $y f_{m}$ has the orbit partition $\mathcal{P}_{[y]}$ on $X$, since $y f_{a m}=\left(y f_{m}\right)^{a}$ for all $a \in \mathbf{Z}$. On the other hand, if $y$ lies in an infinite orbit of $h$ then $\mathcal{P}_{[y]}$ is the partition of $X$ into singleton parts, which is the orbit partition of the identity of $G$. So (2) and (3) are necessary.

Now suppose that the stated conditions hold. We shall construct a permutation $k \in G$ ? $H$ such that $\pi(k)=\mathcal{P}$. By (1) there exists an element $h$ of $H$ whose orbit partition is $\tilde{\mathcal{P}}$. Let $\left\{y_{i} \mid i \in I\right\}$ be a set of representatives for the orbits of $h$, where $I$ is a suitable indexing set. Let $m_{i} \in \mathbf{N} \cup\{\infty\}$ be the size of the orbit containing $y_{i}$. By (4) there exists $c_{(i, t)} \in G$ for $i \in I$ such that

$$
\left(x, y_{i} h^{t}\right) \equiv_{\mathcal{P}}\left(x c_{(i, t)}, y_{i} h^{t+1}\right)
$$

for all $x \in X$ and $t \in \mathbf{Z}$.

Suppose that $m_{i}<\infty$. Then the element

$$
b_{i}=c_{(i, 0)} c_{(i, 1)} \cdots c_{\left(i, m_{i}-1\right)}
$$

of $G$ stabilizes the partition $\mathcal{P}_{\left[y_{i}\right]}$ of $X$. It is possible that $\pi\left(b_{i}\right)$ is a strict refinement of $\mathcal{P}_{\left[y_{i}\right]}$. However, by condition (2), there exists $g_{i} \in G$ such that $\pi\left(g_{i}\right)=\mathcal{P}_{\left[y_{i}\right]}$; if we replace $c_{(i, 0)}$ with $g_{i} b_{i}^{-1} c_{(i, 0)}$, we then have $b_{i}=g_{i}$ and so 
$\pi\left(b_{i}\right)=\mathcal{P}_{\left[y_{i}\right]}$. We may therefore suppose that the $c_{(i, t)}$ have been chosen so that $\pi\left(b_{i}\right)=\mathcal{P}_{\left[y_{i}\right]}$ for all $i$ such that $m_{i}<\infty$.

Let $f \in G^{Y}$ be defined by $\left(y_{i} h^{t}\right) f=c_{(i, t)}$ for each $i \in I$, where $t \in$ $\left\{0, \ldots, m_{i}-1\right\}$ if $m_{i}<\infty$ and $t \in \mathbf{Z}$ otherwise. Let $k=f h \in G \imath H$. We shall show that $\pi(k)=\mathcal{P}$.

We define

$$
b_{(i, t)}= \begin{cases}c_{(i, 0)} \cdots c_{(i, t-1)} & \text { if } t \geq 0 \\ c_{(i,-1)}^{-1} \cdots c_{(i, t)}^{-1} & \text { if } t<0\end{cases}
$$

and note that

$$
\left(x, y_{i}\right) k^{t}=\left(x b_{(i, t)}, y_{i} h^{t}\right)
$$

for all $t \in \mathbf{Z}$. In particular, if $m_{i}<\infty$, then since $b_{\left(i, m_{i}\right)}=g_{i}$, we have $\left(x, y_{i}\right) k^{m_{i}}=\left(x g_{i}, y_{i}\right)$. Observe also that, by $(\star)$, we have

$$
\left(x, y_{i}\right) \equiv_{\mathcal{P}}\left(x b_{(i, t)}, y_{i} h^{t}\right)
$$

for all $t \in \mathbf{Z}$.

Suppose that $\left(x_{1}, y_{i} h^{s}\right) \equiv_{\mathcal{P}}\left(x_{2}, y_{i} h^{t}\right)$. Then by the observation just made we have

$$
\begin{aligned}
& \left(x_{1}, y_{i} h^{s}\right) \equiv_{\mathcal{P}}\left(x_{1} b_{(i, s)}^{-1}, y_{i}\right) \\
& \left(x_{2}, y_{i} h^{t}\right) \equiv_{\mathcal{P}}\left(x_{2} b_{(i, t)}^{-1}, y_{i}\right) .
\end{aligned}
$$

Hence

$$
x_{1} b_{(i, s)}^{-1} \equiv_{\mathcal{P}_{\left[y_{i}\right]}} x_{2} b_{(i, t)}^{-1} .
$$

If $m_{i}<\infty$, then since $\pi\left(b_{\left(i, m_{i}\right)}\right)=\pi\left(g_{i}\right)=\mathcal{P}_{\left[y_{i}\right]}$, the elements $\left(x_{1} b_{(i, s)}^{-1}, y_{i}\right)$ and $\left(x_{2} b_{(i, t)}^{-1}, y_{i}\right)$ lie in the same orbit of $k^{m_{i}}$. Therefore

$$
\left(x_{1}, y_{i} h^{s}\right) k^{-s}=\left(x_{1} b_{(i, s)}^{-1}, y_{i}\right) \equiv_{\pi(k)}\left(x_{2} b_{(i, t)}^{-1}, y_{i}\right)=\left(x_{2}, y_{i} h^{t}\right) k^{-t} .
$$

On the other hand, if $m_{i}=\infty$, then by (3), $\mathcal{P}_{\left[y_{i}\right]}$ is the partition of $X$ into singleton sets and so $x_{1} b_{(i, s)}^{-1}=x_{2} b_{(i, t)}^{-1}$. Therefore $(\dagger)$ also holds in this case. It follows that

$$
\left(x_{1}, y_{i} h^{s}\right) \equiv_{\pi(k)}\left(x_{2}, y_{i} h^{t}\right)
$$

and so $\mathcal{P} \preccurlyeq \pi(k)$.

The argument of the previous paragraph in reverse, again using $(\dagger)$, implies that $\pi(k) \preccurlyeq \mathcal{P}$, and so we have equality as required.

With Lemma 5.1, we are now in a position to prove the following result, which combined with Proposition 2.4 completes the proof of Theorem 2(b).

Proposition 5.2. Suppose that $G$ and $H$ are join-coherent permutation groups on $X$ and $Y$ respectively, and that $Y$ is finite. Then $G \curlywedge H$ is joincoherent in its imprimitive action on $X \times Y$. 
Proof. Let $f_{1} h_{1}$ and $f_{2} h_{2}$ be elements of $G \imath H$, and let $K$ be the subgroup they generate. Let $\mathcal{P}$ be the partition of $X \times Y$ into the orbits of $K$. It suffices to show that the conditions stated in Lemma 5.1 are satisfied by $\mathcal{P}$. Notice that condition (3) is satisfied vacuously, since $Y$ is supposed to be finite.

Since $H$ is join-coherent, it has an element $h$ whose orbit partition $\mathcal{S}$ on $Y$ is the same as that of the subgroup $\left\langle h_{1}, h_{2}\right\rangle$. It is easy to see that $\tilde{\mathcal{P}}=\mathcal{S}$, and so (1) is satisfied.

For $y \in Y$ let $B_{y}=X \times\{y\}$; as noted at the start of this section, $\left\{B_{y} \mid y \in Y\right\}$ is a system of imprimitivity for $G$ 乙 $H$ on $X \times Y$. We write $R_{y}$ for the set-wise stabilizer of $B_{y}$ in $G$ 乙 $H$. In the action of $R_{y}$ on $X$ inherited from the action of $R_{y}$ on $B_{y}$, an element $f h \in R_{y}$ acts on $X$ as $y f \in G$. Thus if $\vartheta: R_{y} \rightarrow G$ is the homomorphism defined by $(f h) \vartheta=y f$, then $\left(K \cap R_{y}\right) \vartheta$ has orbit partition $\mathcal{P}_{[y]}$. Now $K \cap R_{y}$ is finitely generated, since it has finite index in $K$. Since $G$ is join-coherent, it follows that there exists an element $c_{y}$ of $G$ whose orbit partition is $\mathcal{P}_{[y]}$. This gives us (2).

Finally suppose that $y$ and $z$ lie in the same part of $\tilde{\mathcal{P}}$. Then there exist $f \in G^{Y}$ and $h \in H$ such that $y h=z$, and such that $f h \in K$. Let $c=y f$. We see that $(x, y) \equiv_{\mathcal{P}}(x c, z)$ for all $x \in X$, and so (4) is satisfied. This completes the proof.

\section{Centralizers}

The first part of this paper ends with the proof of Theorem 3. The structure of centralizers in symmetric groups is well known.

Lemma 6.1. Let $\Omega$ be a set, let $G=\operatorname{Sym}(\Omega)$, and let $g \in G$. For $k \in$ $\mathbf{N} \cup\{\infty\}$, let $\Delta_{k}$ be the set of orbits of $g$ of size $k$. Then as permutation groups, we have

$$
\operatorname{Cent}_{G}(g)=\prod_{k \in \mathbf{N} \cup\{\infty\}}\left(C_{k} 2 \operatorname{Sym}\left(\Delta_{k}\right)\right),
$$

where $C_{\infty}$ is understood to be $\mathbf{Z}$, the wreath products take the imprimitive action, and the factors in the direct product act on disjoint sets.

With notation as in Lemma 6.1, suppose that $g \in G$ has finitely many orbits of all sizes $k \geq 2$, including $k=\infty$. Then the join-coherence of $\operatorname{Cent}_{G}(g)$ follows immediately from Lemma 6.1 using Propositions 4.2(1) and 5.2. This is sufficient to establish Theorem 3 so far as join-coherence is concerned. However it tells us nothing about meet-coherence, for which we require an account of orbit partitions in the centralizers of semiregular permutations. 
Lemma 6.2. Let $\Omega$ be a set and let $G=\operatorname{Sym}(\Omega)$. Let $\mathcal{P}$ be a partition of $\Omega$, and let $g \in G$ be an element such that $\langle g\rangle$ acts semiregularly on $\Omega$. There exists $h \in \operatorname{Cent}_{G}(g)$ with orbit partition $\mathcal{P}$ if and only if the following conditions hold:

(1) $\mathcal{P}^{g}=\mathcal{P}$;

(2) every part of $\mathcal{P}$ is countable;

(3) if $P$ is an infinite part of $\mathcal{P}$, then either $P$ meets only finitely many $g$ orbits, or else the elements of $P$ lie in distinct $g$ orbits.

If $m \in \mathbf{N} \cup\{\infty\}$ is the order of the semiregular permutation $g$, and $\Delta$ is the set of orbits of $g$, then by Lemma 6.1, we have $\operatorname{Cent}_{G}(g) \cong C_{m} \imath \operatorname{Sym}(\Delta)$, in its imprimitive action. Using this, it is possible to prove Lemma 6.2 by showing the equivalence of its conditions with those of Lemma 5.1, However we prefer to give an independent and more illuminating proof in which we explicitly construct an element of $\operatorname{Cent}_{G}(g)$ whose orbit partition is $\mathcal{P}$.

Proof. It is clear that the first two conditions are necessary. To see that the third condition is also necessary, suppose that $\mathcal{P}$ is the orbit partition of $h \in \operatorname{Cent}_{G}(g)$. Let $P$ be a part of $\mathcal{P}$, and let $x \in P$. Since $h$ stabilizes the orbit partition of $g$, the set $A=\left\{n \in \mathbf{Z} \mid x h^{n} \equiv_{\pi(g)} x\right\}$ is a subgroup of $\mathbf{Z}$. The index $|\mathbf{Z}: A|$ is equal to the number of $g$-orbits represented in $P$; if this number is infinite then $A=\{0\}$, since $\mathbf{Z}$ has no other subgroup of infinite index.

Now suppose that the stated conditions hold. We shall construct an element $h \in \operatorname{Cent}_{G}(g)$ whose orbit partition is $\mathcal{P}$. Let $\left\{P_{i} \mid i \in I\right\}$ be a set of orbit representatives for the action of $g$ on the parts of $\mathcal{P}$, where $I$ is a suitable indexing set. Let $S_{i}=\bigcup_{j \in \mathbf{Z}} P_{i} g^{j}$. It is clear that the sets $S_{i}$ form a partition $\mathcal{S}$ of $\Omega$. In fact it is easy to see that $\mathcal{S}=\mathcal{P} \vee \pi(g)$.

Since $h$ may be defined separately on the distinct parts of $\mathcal{S}$, we may suppose without loss of generality that $\mathcal{S}$ is the trivial partition of $\Omega$ into a single part.

Let $P$ be a part of $\mathcal{P}$, and let $X=\left\{x_{j} \mid j \in J\right\} \subseteq P$ be a set of representatives for the orbits of $g$ on $\Omega$, where $J$ is a suitable indexing ordinal. If $J$ is infinite then it may be taken to be the smallest infinite ordinal $\omega$. By assumption $P$ is countable, and either $J$ is finite or else $X=P$. Let $t$ be the least positive integer such that $P g^{t}=P$, or 0 if no such integer exists. It is clear that if $t=0$ then $X=P$.

We have assumed also that $g$ is a semiregular permutation. Let $m$ be the length of a cycle of $g$; here $m$ may be infinite. For convenience we define $M=\mathbf{Z} / m \mathbf{Z}$ if $m$ is finite, and $M=\mathbf{Z}$ if $m$ is infinite. We shall allow $g$ to 
take exponents from $M$. Every element of $\Omega$ has a unique representation as $x_{j} g^{k}$ for some $j \in J$ and $k \in M$.

Observe that $x_{j} g^{k}$ lies in $P$ if and only if $k$ is a multiple of $t$. Define $h$ on $\Omega$ by

$$
x_{j} g^{k} h= \begin{cases}x_{j+1} g^{k} & \text { if } j+1<J \\ x_{0} g^{k+t} & \text { if } j+1=J .\end{cases}
$$

(The second line of the definition, of course, arises only when $J$ is finite.) Thus $h$ fixes each part of $\mathcal{P}$, and permutes the orbits of $g$.

We now show that $h$ commutes with $g$. It suffices to show that $x_{j} g^{k}$ has the same image under $g h$ and under $h g$, for $j \in J$ and $k \in M$. Suppose that $j+1<J ;$ then

$$
\left(x_{j} g^{k}\right) g h=x_{j} g^{k+1} h=x_{j+1} g^{k+1}=x_{j+1} g^{k} g=\left(x_{j} g^{k}\right) h g .
$$

For the remaining case, we have

$$
\left(x_{J-1} g^{k}\right) g h=x_{J-1} g^{k+1} h=x_{0} g^{k+1+t}=x_{0} g^{k+t} g=\left(x_{J-1} g^{k}\right) h g .
$$

It is clear that the points $x_{j}$ lie in a single orbit $\mathcal{O}$ of $h$ and so $X \subseteq \mathcal{O} \subseteq P$. If $J$ is infinite then clearly $\mathcal{O}=P$, since $X=P$. If $J$ is finite then $\mathcal{O}$ contains $x_{j} g^{k}$ for each $j \in J$ and each $k \in M$ such that $k$ is a multiple of $t$; since every element of $P$ is of this form, we have $\mathcal{O}=P$ in this case too. Now since $g$ and $h$ commute, every other part of $\mathcal{P}$ is also an orbit of $h$, and so $\pi(h)=\mathcal{P}$ as required.

We are now in a position to prove Theorem 3, which we restate for convenience.

Theorem 3. Let $\Omega$ be a set, let $G=\operatorname{Sym}(\Omega)$, and let $g \in G$. For $k \in \mathbf{N} \cup\{\infty\}$ let $n_{k}$ be the number of orbits of $g$ of size $k$.

(a) $\operatorname{Cent}_{G}(g)$ is meet-coherent.

(b) If $n_{k}$ is finite for all $k \neq 1$, including $k=\infty$, then $\operatorname{Cent}_{G}(g)$ is joincoherent.

Proof. By Lemma 6.1, we see that $\operatorname{Cent}_{G}(g)$ is the direct product of centralizers of semiregular permutations. By the two parts of Proposition 4.2. it will be sufficient to prove the result in the case that $g$ acts semiregularly on $\Omega$. We therefore suppose that $g$ is a product of cycles of length $k$, where $k \in \mathbf{N} \cup\{\infty\}$.

Let $\mathcal{P}$ and $\mathcal{Q}$ be partitions in $\operatorname{Cent}_{G}(g)$. By Lemma 6.2 we have $\mathcal{P}^{g}=\mathcal{P}$ and $\mathcal{Q}^{g}=\mathcal{Q}$, from which it follows easily that $(\mathcal{P} \vee \mathcal{Q})^{g}=\mathcal{P} \vee \mathcal{Q}$ and $(\mathcal{P} \wedge \mathcal{Q})^{g}=\mathcal{P} \wedge \mathcal{Q}$. It is clear that $\mathcal{P} \wedge \mathcal{Q}$ also satisfies conditions (2) and (3) of Lemma 6.2. and so $\mathcal{P} \wedge \mathcal{Q} \in \pi\left(\operatorname{Cent}_{G}(g)\right)$. Hence $\pi\left(\operatorname{Cent}_{G}(g)\right)$ is meetcoherent. 
Each part of $\mathcal{P}$ and each part of $\mathcal{Q}$ is countable, and so the parts of $\mathcal{P} \vee \mathcal{Q}$ are countable. If $k>1$, then by hypothesis $g$ has only finitely many orbits; therefore the only case in which a part $S$ of $\mathcal{P} \vee \mathcal{Q}$ can meet an infinite number of orbits is when $k=1$, and clearly in this case $S$ meets each orbit in at most one point. Hence $\mathcal{P} \vee \mathcal{Q}$ also satisfies conditions (2) and (3) of Lemma 6.2, and so $\mathcal{P} \vee \mathcal{Q} \in \pi\left(\operatorname{Cent}_{G}(g)\right)$. Hence $\pi\left(\operatorname{Cent}_{G}(g)\right)$ is join-coherent.

We have seen that the condition that the orbit multiplicities $n_{k}$ are finite for $k>1$ is a sufficient condition for the centralizer in $\operatorname{Sym}(\Omega)$ of $g$ to be join-coherent. Our next proposition shows that this is also a necessary condition.

Proposition 6.3. Let $\Omega$ be a set and let $G=\operatorname{Sym}(\Omega)$. Let $g \in G$ be a permutation which has an infinite number of cycles of length $k$, for some $k>1$, where $k$ may be infinite. Then $\operatorname{Cent}_{G}(g)$ is not join-coherent.

Proof. We may assume that $g$ is semiregular, and that all of its orbits have size $k$. We suppose for simplicity that $\Omega$ is countable; the generalization to higher cardinalities is straightforward. Let $S$ be a set of representatives for the orbits of $g$. We define $\mathcal{S}$ by

$$
\mathcal{S}=\left\{S^{g^{i}} \mid i \in M\right\}
$$

where $M=\{0, \ldots, k-1\}$ if $k<\infty$ and $M=\mathbf{Z}$ if $k=\infty$.

It is easy to see that $\mathcal{S}^{g}=\mathcal{S}$. By assumption each part of $\mathcal{S}$ is countable, and it is clear that each part of $\mathcal{S}$ has a single element in common with each orbit of $g$. Hence $\mathcal{S}$ satisfies the conditions of Lemma 6.2, and so $\mathcal{S} \in \pi\left(\operatorname{Cent}_{G}(g)\right)$. However $\pi(g) \vee \mathcal{S}$ is the trivial partition of $\Omega$ into a single part. Since $k>1$, this partition does not satisfy condition (3) of Lemma 6.2. and so $\pi\left(\operatorname{Cent}_{G}(g)\right)$ is not join-coherent.

\section{Frobenius groups}

We recall that a Frobenius group is a transitive permutation group $G$ on a finite set $\Omega$, such that each point stabilizer in $G$ is non-trivial, but the intersection of the stabilizers of distinct points is trivial. The fixed-point free elements of $G$, together with the identity of $G$, form a normal subgroup $K$, called the Frobenius kernel. The Frobenius kernel acts regularly, and it is often useful to identify $K$ with $\Omega$ by fixing an element $\omega \in \Omega$ and mapping $\omega k \in \Omega$ to $k \in G$. The stabilizer $H$ of a point $\omega \in \Omega$ is called a Frobenius complement, and acts semiregularly on $\Omega \backslash\{\omega\}$. Identifying $\Omega$ with $K$ one finds that any complement $H$ embeds into $\operatorname{Aut}(K)$, and so $G$ is isomorphic 
as a permutation group to $K \rtimes H$, where $K$ has the right regular action on itself. (For further results on Frobenius groups the reader is referred to Theorem 10.3 .1 of [7].)

We give a complete account of join- and meet-coherence in Frobenius groups. As well as being a natural object of study, these results will be important in Sections 9 and 10

Proposition 7.1. A Frobenius group is meet-coherent if and only if it is dihedral of prime degree.

Proof. Let $D$ be the dihedral group of prime degree $p$ acting on a $p$-gon $\Pi$. For any vertices $\alpha$ and $\beta$ of $\Pi$, there is a unique reflection mapping $\alpha$ to $\beta$. It follows that if $\mathcal{P}$ and $\mathcal{Q}$ are the orbit partitions of distinct reflections then $\mathcal{P} \wedge \mathcal{Q}$ is the discrete partition. Since the only non-identity elements of $D$ are reflections and full cycles, we see that $D$ is meet-coherent.

For the converse, let $G$ be a meet-coherent Frobenius group with Frobenius kernel $K$, acting on a set $\Omega$. If $g$ and $h$ lie in different point stabilizers, then since $\pi(g) \wedge \pi(h)$ has two singleton parts, we see that $\pi(g) \wedge \pi(h)$ is the orbit partition of the identity. It follows that if $\mathcal{O}_{g}$ is an orbit of $g$, and $\mathcal{O}_{h}$ is an orbit of $h$, then $\left|\mathcal{O}_{g} \cap \mathcal{O}_{h}\right| \leq 1$.

Suppose that $|G: K|>2$. Let $\alpha$ and $\beta$ be distinct points in $\Omega$. Since the point stabilizers of $\alpha$ and $\beta$ meet every coset of $K$, and since there are at least three such cosets, we may choose elements $g \in \operatorname{Stab}_{G}(\alpha)$ and $h \in \operatorname{Stab}_{G}(\beta)$, such that $g h \notin K$. Then $g h$ fixes a point $\gamma \in \Omega$, distinct from $\alpha$ and $\beta$. Now clearly $\{\gamma, \gamma g\}$ is a subset of a $g$-orbit $\mathcal{O}_{g}$, and $\{\gamma g, \gamma g h\}$ is a subset of an $h$-orbit $\mathcal{O}_{h}$. But since $\gamma g h=\gamma$, we have found two points in $\mathcal{O}_{h} \cap \mathcal{O}_{g}$. This contradicts the observation above that $\left|\mathcal{O}_{g} \cap \mathcal{O}_{h}\right| \leq 1$.

Therefore $|G: K|=2$, and if $t \in G \backslash K$, then $t$ acts on $K$ as a fixed-point free automorphism of order 2. It is well-known that this implies that $K$ is an abelian group of odd order, and that $t$ acts by inversion (see [7, Ch. 10, Theorem 1.4]).

Suppose that $K$ has a non-trivial proper subgroup $L$. Since $L$ has odd order, we have $[K: L] \geq 3$. If $k \in K$ then the orbits of $k$ are the cosets of $K$, whereas $t$ preserves $K$ and acts non-trivially on the cosets $K / L$. It easily follows that $\pi(k) \wedge \pi(t)$ is not the orbit partition of any permutation in $G$; this contradicts the assumption that $G$ is meet-coherent. Therefore $K$ is cyclic of prime order $p$, and $G$ is a dihedral group of order $2 p$.

Since a transitive join-coherent permutation group $G$ of finite degree contains a full cycle, it is clear that if $G$ is a Frobenius group then its kernel is 
cyclic. For this reason, we provide a description of Frobenius groups with a cyclic kernel.

Lemma 7.2. Let $n \in \mathbf{N}$, and let $H$ be a non-trivial finite group. There exists a Frobenius group with cyclic kernel $K \cong C_{n}$ and complement $H$ if and only if $H \cong C_{r}$, where $r$ divides $p-1$ for each prime divisor $p$ of $n$.

Proof. Suppose that there exists such a Frobenius group. As mentioned at the start of this section, we may consider $H$ as a group of automorphisms of $K$. Note that any non-identity element of $H$ acts without fixed points on the non-identity elements of $K$. It follows that $n$ is odd, since an even cyclic group has a unique element of order 2 . Let $p$ be a prime divisor of $n$ and let $L \cong C_{p}$ be the unique subgroup of $K$ of order $p$. Then $H$ acts faithfully on $L$ and since $\operatorname{Aut}(L) \cong C_{p-1}$, it follows that $H$ is cyclic of order dividing $p-1$. Hence the order of $H$ divides $p-1$ for each prime divisor $p$ of $n$.

Conversely, let $n=p_{1}^{a_{1}} \ldots p_{m}^{a_{m}}$, let $K=\langle x\rangle \cong C_{n}$, and let $r$ divide $p_{i}-1$ for all $i$. The Chinese Remainder Theorem allows us to choose $d \in \mathbf{N}$ such that $d$ has multiplicative order $r$ modulo $p_{i}^{a_{i}}$ for all $i$. The map $h: x \mapsto x^{d}$ is an automorphism of $K$ of order $r$, and it is easy to check that $H=\langle h\rangle$ is a Frobenius complement for $K$.

We are now in a position to prove the following.

Proposition 7.3. A Frobenius group is join-coherent if and only if it has prime degree.

Proof. Let $G$ be a Frobenius group with kernel $K$ and complement $H$. We identify the set on which $G$ acts with $K$.

Suppose that $G$ has prime degree $p$, and so $K \cong C_{p}$. Let $X$ be a subgroup of $G$. If $K \leq X$ then $X$ is transitive, and its orbit partition is that of a generator of $K$. Otherwise $X \cap K$ is trivial, and since $G / K$ is abelian by Lemma 7.2, it follows that $X$ is also abelian. If $x$ is a non-identity element of $X$, then $x$ fixes a unique element $a$ of $K$; since $X$ centralizes $x$ we have $X \leq \operatorname{Stab}_{G}(a)$. By Proposition 2.3(3) the point stabilizers in $G$ act joincoherently on $K$, and so there exists $h \in \operatorname{Stab}_{G}(a)$ such that the orbit partition of $X$ is $\pi(h)$. Therefore $G$ itself is join-coherent, as required.

For the converse implication, we observe that the non-identity elements of $K$ are precisely the elements of $G$ whose orbit partition has no singleton parts. Hence if $G$ is join-coherent then $\{\pi(k) \mid k \in K\}$ is closed under taking joins. Since the action of $G$ on $K$ is regular, it follows from Proposition 3.3 that $K$ is cyclic.

Suppose for a contradiction that $|K|$ is composite. Then $K$ has a characteristic non-trivial proper cyclic subgroup $\langle k\rangle$. The orbit partition $\pi(k)$ 
is simply the partition of $K$ into the cosets of $\langle k\rangle$. Let $h$ be a non-identity element of $H$. By Lemma 7.2 we see that $|H|$ is coprime with $|K|$, and hence with $|\langle k\rangle|$. Since $H$ acts semiregularly on $K \backslash\{1\}$, no proper coset of $\langle k\rangle$ in $K$ can be a union of orbits of $h$. Now consider the partition $\pi(k) \vee \pi(h)$. It has one part equal to $\langle k\rangle$ itself, and every other part is a union of two or more cosets of $\langle k\rangle$. But this partition cannot be in $\pi(G)$, since it has parts of different sizes, but no singleton parts. Hence $G$ is not join-coherent.

\section{JOIN-COHERENCE IN LINEAR GROUPS}

In this section we let $V$ be a vector space of dimension $d$ over a field $K$. We shall write $\Lambda$ for the set of lines in $V$. Let $G$ be a group in the range $\mathrm{SL}(V) \leq G \leq \mathrm{GL}(V)$. Then $G$ has a natural action on the non-zero points of $V$, and the quotient $G / Z(G)$ acts on $\Lambda$. Since the lines of $V$ form a system of imprimitivity for the action of $G$, we see by Proposition 2.4(1) that if $G$ acts join-coherently then so does $G / Z(G)$. The main results of this section, Proposition 8.6 and Proposition 8.7, show that if $d>1$ then these actions are almost never join-coherent. These results are used in our classification of join-coherent primitive groups in Section 9 ,

When $d=1$ we see that $G$ is a cyclic group acting semiregularly, and so the action is join-coherent; of course the group $G / Z(G)$ is trivial in this case. When $d>1$ we shall see that it is possible to reduce to the case when $V$ is a 2-dimensional space; in this case we identify $\Lambda$ with the set $K \cup\{\infty\}$, by identifying the line through $(a, b) \in K^{2}$ with $b / a$ when $a \neq 0$, and with $\infty$ when $a=0$. The group $\mathrm{PGL}_{2}(K)$ acting on $\Lambda$ may then be identified with the group of fractional linear transformations

$$
\alpha \mapsto \frac{a \alpha+b}{c \alpha+d}, \quad a, b, c, d \in K, a d-b c \neq 0 .
$$

The following fact is well known.

Lemma 8.1. The action of the group $\mathrm{PGL}_{2}(K)$ on $\Lambda$ is sharply 3 -transitive.

We shall write $K_{0}$ for the characteristic subfield of $K$, and $\Lambda_{0}$ for the subset $K_{0} \cup\{\infty\}$ of $\Lambda$. Our next proposition establishes that $\mathrm{PGL}_{2}(K)$ and $\mathrm{PSL}_{2}(K)$ are join-coherent only in a few small cases.

Lemma 8.2. Let $K$ be a field, and let $G$ be in the range $\mathrm{SL}_{2}(K) \leq G \leq$ $\mathrm{GL}_{2}(K)$. Then $G / Z(G)$ is join-coherent in its action on $\Lambda$ if and only if $G=\mathrm{GL}_{2}\left(\mathbf{F}_{2}\right)$ or $G=\mathrm{GL}_{2}\left(\mathbf{F}_{3}\right)$.

Proof. Suppose first of all that $G$ contains elements of determinant -1 . (This is always the case if char $K=2$.) Then $\mathrm{PGL}_{2}(K)$ has a subgroup isomorphic 
to $S_{3}$, generated by elements $g$ and $h$ which act on $\Lambda$ as $g: \alpha \mapsto 1 / \alpha$ and $h: \alpha \mapsto 1-1 / \alpha$. The parts of $\pi(g) \vee \pi(h)$ have the form

$$
\{\alpha, 1 / \alpha, 1-\alpha, 1-1 / \alpha, \alpha /(\alpha-1),(\alpha-1) / \alpha\}
$$

for $\alpha \in K$. One part is $\{0,1, \infty\}$. If char $K=3$ then $\{-1\}$ is a singleton part, and if char $K \geq 5$ then $\{-1,2,1 / 2\}$ is a part of size 3 . If $K$ contains primitive cube roots of 1 then they form a part of size 2. All other parts have size 6. If $k \in \mathrm{PGL}_{2}(K)$ has orbit partition $\pi(g) \vee \pi(h)$ then $k^{3}$ has at least 3 fixed points; since $\mathrm{PGL}_{2}(K)$ is sharply 3-transitive, it follows that $k^{3}=1$. Therefore $|K| \leq 3$. The well-known isomorphisms $\mathrm{PGL}_{2}\left(\mathbf{F}_{2}\right) \cong S_{3}$ and $\mathrm{PGL}_{2}\left(\mathbf{F}_{3}\right) \cong S_{4}$ now give the join-coherent groups appearing in the lemma.

To deal with the remaining case it will be useful to observe that if $k \in$ $\mathrm{PGL}_{2}(K)$ fixes $\Lambda_{0}$ set-wise then there exist $x, y, z \in \Lambda_{0}$ whose images under $k$ are $0,1, \infty$, respectively; since $\mathrm{PGL}_{2}(K)$ is sharply 3 -transitive it follows that $k$ is the map

$$
\alpha \longmapsto \frac{(y-z)(\alpha-x)}{(y-x)(\alpha-z)},
$$

and so $k \in \mathrm{PGL}_{2}\left(K_{0}\right)$.

Suppose that char $K>0$ and that $G$ has no elements of determinant -1 . It is clear that $G / Z(G)$ acts transitively on $\Lambda_{0}$. Suppose that $t \in G$ has a single orbit on $\Lambda_{0}$. Then $t$ lies in the subgroup of $\mathrm{GL}_{2}\left(\mathbf{F}_{p}\right)$ generated by a Singer element $s \in \mathrm{GL}_{2}\left(\mathbf{F}_{p}\right)$ of order $p^{2}-1$. The determinant of $s$ is a generator of $\mathbf{F}_{p}^{\times}$and hence $(\operatorname{det} s)^{(p-1) / 2}=-1$. By assumption $G$ has no elements of determinant -1 , and so if $p-1=2^{a} c$ where $c$ is odd, then $t \in\left\langle s^{2^{a}}\right\rangle$. However, it is clear that $s$ acts as a $(p+1)$-cycle on $\Lambda_{0}$, and so $s^{2}$ has two orbits on $\Lambda_{0}$. Hence no such element $t$ can exist, and so $G$ is not join-coherent in its action on $\Lambda$.

Finally suppose that char $K=0$. It is easy to show that $G$ contains elements $g$ and $h$ such that $\alpha g=4 \alpha$, and $\alpha h=9 \alpha$ for all $\alpha \in K$. (Here 4 and 9 may be replaced with any two squares that generate a non-cyclic subgroup of $\mathbf{Q}^{\times}$.) Suppose that $t \in G$ is such that $\pi(t)=\pi(g) \vee \pi(h)$. Since $t$ has both 0 and $\infty$ as fixed points, it easily follows that there exists $x \in \mathbf{Q}$ such that $\alpha t=x \alpha$ for all $\alpha \in K$. The orbit of $\langle g, h\rangle$ on $\mathbf{Q} \cup\{\infty\}$ containing 1 is $\left\{4^{i} 9^{j}: i, j \in \mathbf{Z}\right\}$, whereas the orbit of $\langle t\rangle$ on $\mathbf{Q} \cup\{\infty\}$ containing 1 is $\left\{x^{i}: i \in \mathbf{Z}\right\}$. It is clear that these sets are not equal for any choice of $x \in \mathbf{Q}$. Hence $G / Z(G)$ is not join-coherent in its action on $\Lambda$.

It is worth noting that $\mathrm{PSL}_{2}(\mathbf{Q})$ does contain finitely generated subgroups that are transitive on $\mathbf{Q} \cup\{\infty\}$. For example, one such subgroup is generated 
by the maps $g: \alpha \mapsto \alpha+1$ and $h: \alpha \mapsto \alpha /(\alpha+1)$ used to define the CalkinWilf tree of rational numbers [3]. It can be shown, however, that no element $k \in \mathrm{PGL}_{2}(\mathbf{Q})$ acts transitively on $\mathbf{Q} \cup\{\infty\}$; this gives an alternative way to conclude the proof of Lemma 8.2 .

Lemma 8.2 is the basis for the following more general statement.

Proposition 8.3. Let $V$ be a vector space of dimension $d$ over a field $K$, where $d \geq 2$. Let $G$ be in the range $\mathrm{SL}(V) \leq G \leq \mathrm{GL}(V)$. Then the action of $G / Z(G)$ on the lines of $V$ is not join-coherent unless $d=2$ and $|K| \leq 3$.

Proof. Let $W$ be a subspace of $V$, and let $G_{W}$ be the set-stabilizer of $W$ in $G$. By Proposition 2.3(2), if $G$ is join-coherent in its projective action on the lines of $V$, then $G_{W}$ is join-coherent on the lines of $W$.

Take $W$ to be a 2-dimensional subspace of $V$. By Lemma 8.2, applied to $G_{W}$, we see that $G$ cannot be join-coherent unless $|K| \leq 3$. Suppose that $|K| \leq 3$ and $d \geq 3$. Take $W$ to be a 3 -dimensional subspace of $V$. The possibilities for $G_{W} / Z\left(G_{W}\right)$ are $\mathrm{PGL}_{3}\left(\mathbf{F}_{2}\right)$ and $\mathrm{PSL}_{3}\left(\mathbf{F}_{3}\right)$ and $\mathrm{PGL}_{3}\left(\mathbf{F}_{3}\right)$. A straightforward computation, using the software mentioned in the introduction, shows that none of these groups is join-coherent, and so if $d \geq 3$ then $G / Z(G)$ is not join-coherent.

Let $\Phi$ be a non-trivial group of automorphisms of the field $K$, and let $G$ be a group such that $\mathrm{SL}(V) \leq G \leq \mathrm{GL}(V)$. Then $G \cdot \Phi$ acts on the non-zero points of $V$, and $(G \cdot \Phi) / Z(G)$ acts on the set $\Lambda$ of lines.

Lemma 8.4. Let $\Phi$ be a non-trivial group of automorphisms of a finite field $K$. Let $G$ be in the range $\mathrm{SL}_{2}(K) \leq G \leq \mathrm{GL}_{2}(K)$. Then $(G \cdot \Phi) / Z(G)$ is join-coherent on $\Lambda$ if and only if $K=\mathbf{F}_{4}, G=\mathrm{SL}_{2}\left(\mathbf{F}_{4}\right)$ or $G=\mathrm{GL}_{2}\left(\mathbf{F}_{4}\right)$, and $\Phi=\operatorname{Gal}\left(\mathbf{F}_{4}: \mathbf{F}_{2}\right)$.

Proof. Let $|K|=p^{r}$ where $p$ is prime. Since $K$ admits non-trivial automorphisms, we must have $r>1$. Let $H$ be the stabilizer in $G \cdot \Phi$ of a distinguished line $\ell \in \Lambda$. Since the action of $\operatorname{PSL}_{2}(K)$ on $\Lambda$ is 2 -transitive, the action of $H$ on $\Lambda \backslash\{\ell\}$ is transitive. If $G \cdot \Phi$ is join-coherent on $\Lambda$, then by Proposition 2.3(3), so is $H$. It follows that $H$ must contain an element of order $p^{r}$. Let $r=p^{a} m$ where $p$ does not divide $m$. Let $g \in G \cdot \Phi$ be a $p$-element. Since the full automorphism group of $K$ has order $r$, we see that $g^{p^{a}} \in G$. But a non-trivial unipotent element of $\mathrm{GL}_{2}(K)$ has order $p$, and so $g^{p^{a+1}}=1$. Hence the order of $g$ is at most $p^{a+1}$. However, $p^{a+1}$ is strictly less than $p^{r}$, except in the case when $a=1, r=2$ and $p=2$, and so $p^{r}=4$.

When $K=\mathbf{F}_{4}$ we observe that since every element in $\mathbf{F}_{4}$ is a square, $\mathrm{PGL}_{2}\left(\mathbf{F}_{4}\right)=\mathrm{PSL}_{2}\left(\mathbf{F}_{4}\right)$. Moreover, $\mathrm{PGL}_{2}\left(\mathbf{F}_{4}\right) \cdot \operatorname{Gal}\left(\mathbf{F}_{4}: \mathbf{F}_{2}\right)$ is isomorphic to 
the symmetric group $S_{5}$, in its standard action on 5 points, and is therefore join-coherent.

The principal difficulty in extending Lemma 8.4 to general fields comes from simple transcendental extensions of $\mathbf{F}_{p}$ for small primes $p$. Let $K=$ $\mathbf{F}_{p}(x)$, where $x$ is a transcendental element. We shall represent elements of $K \cup\{\infty\}$ as rational quotients $P(x) / Q(x)$, where not both $P(x)$ and $Q(x)$ are zero, taking the quotients in which $Q(x)=0$ to represent $\infty$. Recalling our earlier identification of $K \cup\{\infty\}$ with the projective line $\Lambda$, this gives a convenient representation for the points of $\Lambda$. In this representation, the fractional linear transformation $\alpha \mapsto(A \alpha+B) /(C \alpha+D)$ in $\mathrm{PGL}_{2}(K)$ acts by

$$
\frac{P(x)}{Q(x)} \mapsto \frac{A P(x)+B Q(x)}{C P(x)+D Q(x)} .
$$

It is straightforward to check that this is a well-defined action of $\mathrm{PGL}_{2}(K)$ on $\Lambda$.

Lemma 8.5. Let $p$ be prime and let $K=\mathbf{F}_{p}(x)$, where $x$ is a transcendental element. Let $\Phi=\operatorname{Gal}\left(K: \mathbf{F}_{p}\right)$ and let $H=\operatorname{PGL}_{2}(K)$. There is an action of $H \cdot \Phi$ on $\Lambda$ defined by

$$
h \varphi: \frac{P(x)}{Q(x)} \rightarrow\left(\frac{P(x \varphi)}{Q(x \varphi)}\right) h .
$$

In this action, $H \cdot \Phi$ acts regularly on the orbit containing $x^{p+1}+x^{p}$.

Proof. The remarks made immediately before the lemma show that the action is well-defined. Let $P(x)=x^{p+1}+x^{p}$. It suffices to show that if $P(x) h=P(x \varphi)$ for $h \in H$ and $\varphi \in \operatorname{Gal}\left(K: \mathbf{F}_{p}\right)$, then $h$ and $\varphi$ are the identities in their respective groups. It is clear that $\varphi$ is determined by its effect on $x$, and it is well known that

$$
x \varphi=\frac{a x+b}{c x+d}
$$

for some $a, b, c, d \in \mathbf{F}_{p}$ with $a d-b c \neq 0$. (In fact $\operatorname{Gal}\left(K: \mathbf{F}_{p}\right) \cong \mathrm{PSL}_{2}\left(\mathbf{F}_{p}\right)$, and so we have two distinct projective linear groups, acting in different ways on $\Lambda$.) Suppose that

$$
P(x \varphi)=\frac{(a x+b)^{p+1}}{(c x+d)^{p+1}}+\frac{(a x+b)^{p}}{(c x+d)^{p}}=\frac{A\left(x^{p+1}+x^{p}\right)+B}{C\left(x^{p+1}+x^{p}\right)+D}=P h .
$$

Then using the fact that $(r x+s)^{p}=r x^{p}+s$ for any $r, s \in K_{0}$, we have

$$
\begin{aligned}
\left(a x^{p}+b\right)((a+c) x+ & (b+d))\left(C x^{p+1}+C x^{p}+D\right) \\
& =\left(c x^{p}+d\right)(c x+d)\left(A x^{p+1}+A x^{p}+B\right) .
\end{aligned}
$$


By comparing the coefficients of $x$ on both sides of this equation, starting with the constant and linear terms, it is now easy to show that $h$ and $\varphi$ are the identity maps.

We are now ready to prove our main result on the action of extended linear groups on lines.

Proposition 8.6. Let $\Phi$ be a non-trivial group of automorphisms of a field $K$. Let $V$ be a d-dimensional space over $K$, and let $G$ be in the range $\mathrm{SL}(V) \leq G \leq \mathrm{GL}(V)$. If $(G \cdot \Phi) / Z(G)$ is join-coherent on the lines of $V$, then $K=\mathbf{F}_{4}, G=\mathrm{SL}_{2}\left(\mathbf{F}_{4}\right)$ or $G=\mathrm{GL}_{2}\left(\mathbf{F}_{4}\right)$, and $\Phi=\operatorname{Gal}\left(\mathbf{F}_{4}: \mathbf{F}_{2}\right)$.

Proof. Let $W_{0} \subseteq V$ be a 2-dimensional vector space over $K_{0}$, and define $W=W_{0} \otimes_{K_{0}} K$. Then $\Phi$ stabilizes $W$ as a set. By Proposition 2.3(2), if $(G \cdot \Phi) / Z(G)$ is join-coherent on the lines of $V$, then the set stabilizer of $W$ is join-coherent on the lines of $W$. Therefore, provided $K \neq \mathbf{F}_{4}$, it is sufficient to prove the theorem in the case $d=2$. By a similar argument, it is sufficient in the case $K=\mathbf{F}_{4}$ to show that the groups $\mathrm{PGL}_{3}(4) \cdot \operatorname{Gal}\left(\mathbf{F}_{4}: \mathbf{F}_{2}\right)$ and $\mathrm{PSL}_{3}(4) \cdot \operatorname{Gal}\left(\mathbf{F}_{4}: \mathbf{F}_{2}\right)$ are not join-coherent. This follows from a straightforward computation.

We shall therefore assume that $V$ is 2-dimensional. We need the following observation: if $E$ is a subfield of $K$ and $H_{E}$ is the set-stabilizer of the set $\Lambda_{E}$ of lines in $\Lambda$ contained in $W_{0} \otimes_{K_{0}} E$, then by Proposition 2.3(2), the action of $H_{E}$ on $\Lambda_{E}$ is join-coherent.

We first use this observation in the case $E=\mathbf{F}_{p}$. Then $\Lambda_{E}=\Lambda_{0}$, and since $\Phi$ acts trivially on $\Lambda_{0}$, we may apply Lemma 8.2 to the group $H_{E} \leq$ $\mathrm{GL}_{2}\left(\mathbf{F}_{p}\right)$ to deduce that $\left|K_{0}\right| \leq 3$. Hence $K_{0}=\mathbf{F}_{p}$ where $p \leq 3$. If $K$ is algebraic over $\mathbf{F}_{p}$ then $K$ is a finite field, and so, by Lemma 8.4, we have $K=\mathbf{F}_{4}$ and $G=\mathrm{SL}_{2}\left(\mathbf{F}_{4}\right)$ or $G=\mathrm{GL}_{2}\left(\mathbf{F}_{4}\right)$.

Now suppose that $K$ is not an algebraic extension of $\mathbf{F}_{p}$. In this case there exists $x \in K$ such that $x$ is transcendental over $\mathbf{F}_{p}$. Applying the observation to the purely transcendental extension $E=\mathbf{F}_{p}(x)$, we see that $H_{E} \cdot \Phi$ acts join-coherently on $\Lambda_{E}$. By Lemma 8.5, the group $H_{E} \cdot \Phi$ has a regular orbit in its action on $K \cup\{\infty\}$. However $H_{E} \cdot \Phi$ is not locally cyclic, and so, by Proposition 3.3, the action of $H_{E} \cdot \Phi$ is not join-coherent.

Finally, we extend the results to the action of $\mathrm{GL}(V)$ on the non-zero points of $V$.

Proposition 8.7. Let $V$ be a d-dimensional vector space over a field $K$, where $d>1$. Let $\Phi$ be a group (possibly trivial) of automorphisms of $K$, and let $G$ be in the range $\mathrm{SL}(V) \leq G \leq \mathrm{GL}(V)$. If $G \cdot \Phi$ is join-coherent in its action on $V \backslash\{0\}$ then $K=\mathbf{F}_{2}$ and $d=2$. 
Proof. The set of punctured lines $\{\ell \backslash\{0\} \mid \ell \in \Lambda\}$ form a system of imprimitivity for the action of $G$ on $V \backslash\{0\}$. Hence, by Proposition 2.4(1), if $G \cdot \Phi$ is join-coherent on points, then $(G \cdot \Phi) / Z(G)$ is join-coherent on lines. It follows from Propositions 8.3 and 8.6 that $G \cdot \Phi$ is one of $\mathrm{GL}_{2}\left(\mathbf{F}_{2}\right), \mathrm{GL}_{2}\left(\mathbf{F}_{3}\right)$ or $\mathrm{GL}_{2}\left(\mathbf{F}_{4}\right) \cdot \operatorname{Gal}\left(\mathbf{F}_{4}: \mathbf{F}_{2}\right)$. A computation shows that the only one of these groups which is join-coherent on points is $\mathrm{GL}_{2}\left(\mathbf{F}_{2}\right)$.

\section{Primitive Join-COHerent Groups of Finite Degree}

In this section we shall establish Theorem 4 , that a primitive permutation group of finite degree is join coherent if and only if it is a symmetric group or a subgroup of the Frobenius group $\mathrm{AGL}_{1}\left(\mathbf{F}_{p}\right)$ in its action on $p$ points.

If $G$ is a primitive join-coherent group of finite degree $n$ then it contains an $n$-cycle. The following lemma, classifying such groups, is Theorem 3 in [9]. (The reader is referred to [9] for original references.) We shall write $\mathrm{P} L_{d}\left(\mathbf{F}_{q}\right)$ for the group $\mathrm{PGL}_{d}\left(\mathbf{F}_{q}\right) \cdot \Phi$, where $\Phi$ is the Galois group of $\mathbf{F}_{q}$ over its prime subfield.

Lemma 9.1. Let $G$ be a primitive permutation group on $n$ points containing an $n$-cycle. Then one of the following holds:

(1) $G$ is $S_{n}$ or $A_{n}$;

(2) $n=p$ is a prime, and $G \leq \operatorname{AGL}_{1}\left(\mathbf{F}_{p}\right)$;

(3) $\mathrm{PGL}_{d}\left(\mathbf{F}_{q}\right) \leq G \leq \mathrm{P \Gamma L}_{d}\left(\mathbf{F}_{q}\right)$ for $d>1$, where $n=\left(q^{d}-1\right) /(q-1)$, the action being either on projective points or on hyperplanes;

(4) $G$ is $\mathrm{PSL}_{2}\left(\mathbf{F}_{11}\right)$ or $M_{11}$ acting on 11 points, or $M_{23}$ acting on 23 points.

It is straightforward to prove Theorem 4 using this lemma and the results of Sections 7 and 8. Certainly $S_{n}$ is join-coherent, and we have seen that $A_{n}$ is not join-coherent when $n>3$. A transitive subgroup of $\operatorname{AGL}_{1}\left(\mathbf{F}_{p}\right)$ is a Frobenius group, and is therefore join-coherent by Proposition 7.3 .

Suppose that $\mathrm{PGL}_{d}\left(\mathbf{F}_{q}\right) \leq G \leq \mathrm{P}_{d}\left(\mathbf{F}_{q}\right)$. The actions of $G$ on points and on hyperplanes are dual to one another, and it therefore suffices to rule out join-coherence for one of them. By Propositions 8.3 and 8.6, the only join-coherent examples in the action on points are $\mathrm{PGL}_{2}\left(\mathbf{F}_{2}\right), \mathrm{PGL}_{2}\left(\mathbf{F}_{3}\right)$ and $\mathrm{P} L_{2}\left(\mathbf{F}_{4}\right)$. As we have seen, these are isomorphic as permutation groups to $S_{3}, S_{4}$ and $S_{5}$ respectively, in their natural actions.

We have therefore reduced the proof to a small number of low degree groups, namely $\mathrm{PSL}_{2}\left(\mathbf{F}_{11}\right)$ in its action on 11 points, and the Mathieu groups $M_{11}$ and $M_{23}$. Establishing that none of these groups is join-coherent is a routine computational task. 


\section{Groups Containing a PROper normal CyCliC SubGroup aCting REGULARLY}

We have observed that a transitive join-coherent permutation group on a finite set must contain a full cycle. We end this paper by investigating the situation when this cycle generates a normal subgroup.

Let $G$ act on $\Omega$, a set of size $n$. Suppose that $K$ is a transitive normal cyclic subgroup of $G$ of order $n$. Let $H$ be the stabilizer of a point $\omega \in \Omega$. Then clearly $G=K \rtimes H$, and by the argument indicated at the start of Section 7 , we may identify $\Omega$ with $K$ by the bijection sending $\omega k \in \Omega$ to $k \in K$. The action of $H$ on $\Omega$ then defines an embedding of $H$ into $\operatorname{Aut}(K)$. Every subgroup of $K$ is characteristic in $K$, and therefore invariant under the action of $H$.

Suppose that $G$ is join-coherent. If $n=a b$ for coprime $a, b$ then $K \cong$ $C_{a} \times C_{b}$, and we see from Proposition 4.4 that $G$ factorizes as $G_{1} \times G_{2}$, where $G_{1}$ is join-coherent on $C_{a}, G_{2}$ is join-coherent on $C_{b}$, and the factors $G_{1}$ and $G_{2}$ have coprime orders. Therefore, to obtain a complete classification, it suffices to consider the case that $n$ is a prime power.

One trivial possibility is that $G=K$; in this case the action of $G$ is semiregular, and join-coherent by Proposition 3.3. If $K$ is assumed to be a proper subgroup, then we shall see that the classification divides into two cases: the case that $n=p$ is prime, and the case that $n=p^{a}$ for $a>1$.

Proposition 10.1. Let $p$ be prime and let $a>1$. Let $\Gamma\left(p^{a}\right)$ be the extension of the additive group $\mathbf{Z} / p^{a} \mathbf{Z}$ by the automorphism $f: x \mapsto r x$, where $r=$ $p^{a-1}+1$. Then $\Gamma\left(p^{a}\right)$ is join-coherent.

Proof. An element $g$ of $\Gamma\left(p^{a}\right)$ may be represented as $x \mapsto r^{j} x+i$ for nonnegative integers $i<p^{a}$ and $j<p$. It is clear that $x g=\left(j p^{a-1}+1\right) x+i$ and so $x g=x$ if and only if $j p^{a-1} x+i=0$. Moreover, a straightforward calculation shows that

$$
x g^{t}-x=t j p^{a-1} x+\frac{t(t-1)}{2} i j p^{a-1}+t i,
$$

and so, if $c \geq 1$, then

$$
x g^{p^{c}}-x= \begin{cases}p^{c} i & \text { if } p \text { is odd } \\ 2^{c} i\left(2^{a-2}\left(2^{c}-1\right) j+1\right) & \text { if } p=2 .\end{cases}
$$

Since $\Gamma\left(p^{a}\right)$ is a $p$-group, and $x g^{t}-x=0$ if and only if the $g$-orbit containing $x$ has size dividing $t$, the preceding equation allows us to determine the sizes of the orbit partitions of elements of $\Gamma\left(p^{a}\right)$. Let $p^{b}$ be the highest power of $p$ dividing $i$ if $i \neq 0$, and let $b=a$ if $i=0$. 
(1) If $b<a-1$, or if $j=0$, then $x g \equiv x \bmod p^{b}$ for all $x \in \mathbf{Z} / p \mathbf{Z}$ and each orbit of $g$ has size $p^{a-b}$. Hence the orbits of $g$ are the cosets of $\left\langle p^{b}\right\rangle$ in $\mathbf{Z} / p^{a} \mathbf{Z}$.

(2) If $b \geq a-1$ and $j \neq 0$, then $x$ is a fixed point of $g$ if and only if $p$ divides $j x+k$, where $i=p^{a-1} k$. Thus the fixed points of $g$ form a coset of $\langle p\rangle$ in $\mathbf{Z} / p^{a} \mathbf{Z}$. The remaining orbits have size $p$ and are cosets of $\left\langle p^{a-1}\right\rangle$ in $\mathbf{Z} / p^{a} \mathbf{Z}$.

From this description of the orbit partitions of elements of $\Gamma\left(p^{a}\right)$, it is not hard to show that $\pi\left(\Gamma\left(p^{a}\right)\right)$ is closed under the join operation.

We remark that when $p$ is odd, the group $\Gamma\left(p^{a}\right)$ is the unique extension of $\mathbf{Z} / p^{a} \mathbf{Z}$ by an automorphism of order $p$. When $p=2$ there are three such extensions, provided $a \geq 3$, of which $\Gamma\left(p^{a}\right)$ is the one which is neither dihedral nor quasidihedral. (There appears to be no widely accepted name for this group.)

Proposition 10.2. Let $p$ be prime, let $a>1$, and let $K$ be the additive group $\mathbf{Z} / p^{a} \mathbf{Z}$. Let $H$ be a non-trivial group of automorphisms of $K$. The group $K \rtimes H$ is join-coherent if and only if it is the group $\Gamma\left(p^{a}\right)$ from Proposition 10.1.

Proof. Let $G$ be the full group of affine transformations of $\mathbf{Z} / p^{a} \mathbf{Z}$. Then $K \rtimes H \leq G$. Let $L$ be the unique subgroup of $K$ of order $p$.

We describe the elements of $G$ which have an orbit equal to $L$. Suppose that $g: x \mapsto r x+s$ is such an element. Then it is easy to see that $s=m p^{a-1}$ for some $m$ not divisible by $p$, since the image of 0 under $g$ is a non-identity element of $L$. Furthermore, since $g$ has no fixed points in $L$, we see that $r \equiv 1 \bmod p$. But these restrictions on $r$ and $s$ imply that the equation $x=r x+s$ has a solution in $\mathbf{Z} / p^{a} \mathbf{Z}$, except in the case that $r=1$, when $g \in L$. Hence $g$ must have a fixed point in $K \backslash L$, except in the case that its orbits are precisely the cosets of $L$ in $K$.

Let $g$ be a generator of $L$. The orbits of $g$ are the cosets of $L$, and the automorphism group $H$ clearly stabilizes $L$ set-wise. It follows that for any $h \in H$, the join $\pi(g) \vee \pi(h)$ has $L$ as a part, and that every part is a union of cosets of $L$. But such a partition has no singleton part, and so cannot be in $\pi(G)$ unless each of its parts is a single coset; this implies that if $K \rtimes H$ is join-coherent, then the action of $H$ on the cosets of $L$ is trivial. It is easy to see that this is the case only if $H=\langle f\rangle$, where $f$ is as in Proposition 10.1.

We are now in a position to prove Theorem 5 . For convenience we restate the theorem below. 
Theorem 5. Let $G$ be a permutation group on $n$ points, containing a normal cyclic subgroup of order $n$ acting regularly. Let $n$ have prime factorization $\prod_{i} p_{i}^{a_{i}}$. Then $G$ is join-coherent if and only if there exists for each $i$ a transitive permutation group $G_{i}$ on $p_{i}^{a_{i}}$ points, such that:

- if $a_{i}>1$ then $G_{i}$ is either cyclic or the extension of a cyclic group of order $p_{i}^{a_{i}}$ by the automorphism $x \mapsto x^{r}$ where $r=p_{i}^{a_{i}-1}+1$,

- if $a_{i}=1$ then $G_{i}$ is a subgroup of the Frobenius group of order $p(p-1)$,

- the orders of the groups $G_{i}$ are mutually coprime,

- $G$ is permutation isomorphic to the direct product of the groups $G_{i}$ in its product action.

Proof. Let $n=\prod_{i} p_{i}^{a_{i}}$, and suppose that $G$ is a join-coherent permutation group on $n$ points containing a regular normal cyclic subgroup $K$ of order $n$. Then we can regard $G$ as acting on $K$. Let $K_{i}$ be the unique subgroup of $K$ of order $p_{i}^{a_{i}}$. Since $K \cong \prod_{i} K_{i}$, it follows easily from Proposition 4.4 that $G \cong$ $\prod_{i} G_{i}$, where $G_{i}$ is the kernel of $G$ in its action on the complement $\prod_{j \neq i} K_{j}$ of $K_{i}$. Moreover Proposition 4.4 implies that the groups $G_{i}$ and $G_{j}$ have coprime orders whenever $i \neq j$, and that $G_{i}$ acts join-coherently on $K_{i}$ for all $i$. If $a_{i}>1$, then by Proposition 10.2, either $G_{i}$ is cyclic of order $p_{i}^{a_{i}}$ or $G_{i}$ is isomorphic to $\Gamma\left(p_{i}^{a_{i}}\right)$, while if $a_{i}=1$ then $G_{i}$ is a subgroup of the normalizer in $S_{p_{i}}$ of a $p_{i}$-cycle, and so is a subgroup of the Frobenius group of order $p_{i}\left(p_{i}-1\right)$. This completes the proof in one direction.

For the converse, suppose that we have for each $i$ a permutation group $G_{i}$ on $p_{i}^{a_{i}}$ points, containing a regular normal cyclic subgroup, and such that if $a_{i}>1$ then $G_{i}$ is either cyclic or isomorphic to $\Gamma\left(p_{i}^{a_{i}}\right)$. If $a_{i}>1$ then Proposition 10.1 tells us that $G_{i}$ is join-coherent. If $a_{i}=1$ on the other hand, then $G_{i}$ is either cyclic or else a Frobenius group of prime degree, in which case it is join-coherent by Proposition 7.3. If the orders of the groups $G_{i}$ are coprime, then their direct product is join-coherent by Proposition 4.3, and this completes the proof.

We end with a remark on the uniqueness of the decomposition in Theorem 5. Since the groups $K_{i}$ and $G_{i}$ appearing in the proof of the theorem are uniquely determined, it follows that any group which satisfies the hypotheses of this theorem has a unique decomposition into a direct product of transitive groups of prime power degrees.

\section{REFERENCES}

[1] Reinhold Baer, Abelian groups without elements of finite order, Duke Math J. 3 (1937), no. 1, 68-122. 
[2] L. Brickman and P. A. Fillmore, The invariant subspace lattice of a linear transformation, Canad. J. Math. 19 (1967), 810-822.

[3] N. Calkin and H. S. Wilf, Recounting the rationals, Amer. Math. Monthly. 107 (200), 360-363.

[4] Peter J. Cameron, Cycle-closed permutation groups, J. Algebraic Combinatorics 5 (1996), 315-322.

[5] Peter J. Cameron, Permutation groups, London Mathematical Society Student Texts 45, Cambridge University Press, 1999.

[6] B.A. Davey and H. A. Priestley, Introduction to Lattices and Order, 2nd ed., Cambridge University Press, 2002.

[7] D. Gorenstein, Finite groups, 2nd ed., Chelsea Publishing Co., New York, 1980.

[8] Wieb Bosma, John Cannon, and Catherine Playoust, The Magma algebra system. I. The user language, J. Symbolic Comput. 24 (1997), 235-265.

[9] G. A. Jones, Cyclic regular subgroups of primitive permutation groups, J. Group Theory 5 (2002), 403-407.

[10] Øystein Ore, Structures and group theory, II, Duke Math. J. 4 (1938), no. 2, 247-269.

Heilbronn Institute for Mathematical Research, School of Mathematics, University of Bristol, University WAlk, Bristol, BS8 1TW

E-mail address: j.r.britnell@bristol.ac.uk

Department of Mathematics, Royal Holloway, University of London, Egham, SURREY TW20 0EX

E-mail address: mark.wildon@rhul.ac.uk 